DOI 10.4171/JEMS/347

Qi Lü · Jiongmin Yong $\cdot$ Xu Zhang

\title{
Representation of Itô integrals by Lebesgue/Bochner integrals
}

Received November 5, 2010 and in revised form March 29, 2011

\begin{abstract}
In [22], it was proved that as long as the integrand has certain properties, the corresponding Itô integral can be written as a (parameterized) Lebesgue integral (or Bochner integral). In this paper, we show that such a question can be answered in a more positive and refined way. To do this, we need to characterize the dual of the Banach space of some vector-valued stochastic processes having different integrability with respect to the time variable and the probability measure. The latter can be regarded as a variant of the classical Riesz Representation Theorem, and therefore it will be useful in studying other problems. Some remarkable consequences are presented as well, including a reasonable definition of exact controllability for stochastic differential equations and a condition which implies a Black-Scholes market to be complete.
\end{abstract}

Keywords. Itô integral, Lebesgue integral, Bochner integral, range inclusion, Riesz-type Representation Theorem

\section{Introduction}

Let $(\Omega, \mathcal{F}, \mathbb{F}, \mathbb{P})$ be a complete filtered probability space with $\mathbb{F}=\left\{\mathcal{F}_{t}\right\}_{t \geq 0}$, on which a one-dimensional standard Brownian motion $\{W(t)\}_{t \geq 0}$ is defined so that $\mathbb{F}$ is its natural filtration augmented by all the $\mathbb{P}$-null sets. Let $H$ be a Banach space with the norm $|\cdot|_{H}$ and with the dual space $H^{*}$. For any $p \in[1, \infty)$, let $L_{\mathcal{F}_{T}}^{p}(\Omega ; H)$ be the set of all $\mathcal{F}_{T^{-}}$ measurable $\left(H\right.$-valued) random variables $\xi: \Omega \rightarrow H$ such that $\mathbb{E}|\xi|_{H}^{p}<\infty$. Next, for any $p, q \in[1, \infty)$, put

$L_{\mathbb{F}}^{p}\left(\Omega ; L^{q}(0, T ; H)\right)=\{\varphi:[0, T] \times \Omega \rightarrow H \mid \varphi(\cdot)$ is $\mathbb{F}$-progressively measurable and

$$
\left.\mathbb{E}\left(\int_{0}^{T}|\varphi(t)|_{H}^{q} d t\right)^{p / q}<\infty\right\},
$$

Q. Lü: School of Mathematical Sciences, University of Electronic Science and Technology of China, Chengdu 610054, China, and School of Mathematics, Sichuan University, Chengdu 610064, China; e-mail: luqi59@163.com

J. Yong: Department of Mathematics, University of Central Florida, FL 32816, USA; e-mail: jyong@mail.ucf.edu

X. Zhang: Yangtze Center of Mathematics, Sichuan University, Chengdu 610064, China, and Key Laboratory of Systems and Control, Academy of Mathematics and Systems Science, Chinese Academy of Sciences, Beijing 100190, China; e-mail: zhang_xu@scu.edu.cn

Mathematics Subject Classification (2010): Primary 60G05; Secondary 60H05, 60G07 
$L_{\mathbb{F}}^{q}\left(0, T ; L^{p}(\Omega ; H)\right)=\{\varphi:[0, T] \times \Omega \rightarrow H \mid \varphi(\cdot)$ is $\mathbb{F}$-progressively measurable and

$$
\left.\int_{0}^{T}\left(\mathbb{E}|\varphi(t)|_{H}^{p}\right)^{q / p} d t<\infty\right\}
$$

In an obvious way, we may also define (for $1 \leq p, q<\infty$ )

$$
\left\{\begin{array}{lll}
L_{\mathbb{F}}^{\infty}\left(\Omega ; L^{q}(0, T ; H)\right), & L_{\mathbb{F}}^{p}\left(\Omega ; L^{\infty}(0, T ; H)\right), & L_{\mathbb{F}}^{\infty}\left(\Omega ; L^{\infty}(0, T ; H)\right), \\
L_{\mathbb{F}}^{\infty}\left(0, T ; L^{p}(\Omega ; H)\right), & L_{\mathbb{F}}^{q}\left(0, T ; L^{\infty}(\Omega ; H)\right), & L_{\mathbb{F}}^{\infty}\left(0, T ; L^{\infty}(\Omega ; H)\right) .
\end{array}\right.
$$

It is clear that

$$
L_{\mathbb{F}}^{p}\left(\Omega ; L^{p}(0, T ; H)\right)=L_{\mathbb{F}}^{p}\left(0, T ; L^{p}(\Omega ; H)\right) \equiv L_{\mathbb{F}}^{p}(0, T ; H), \quad 1 \leq p \leq \infty .
$$

Also, by Minkowski's inequality,

$$
\begin{cases}L_{\mathbb{F}}^{p}\left(\Omega ; L^{q}(0, T ; H)\right) \subseteq L_{\mathbb{F}}^{q}\left(0, T ; L^{p}(\Omega ; H)\right), & 1 \leq p \leq q \leq \infty \\ L_{\mathbb{F}}^{q}\left(0, T ; L^{p}(\Omega ; H)\right) \subseteq L_{\mathbb{F}}^{p}\left(\Omega ; L^{q}(0, T ; H)\right), & 1 \leq q \leq p \leq \infty\end{cases}
$$

In particular,

$$
L_{\mathbb{F}}^{1}\left(0, T ; L^{p}(\Omega ; H)\right) \subseteq L_{\mathbb{F}}^{p}\left(\Omega ; L^{1}(0, T ; H)\right), \quad 1 \leq p \leq \infty .
$$

We now introduce two linear operators

$$
\left\{\begin{array}{l}
\mathbb{I}: L_{\mathbb{F}}^{1}\left(\Omega ; L^{2}(0, T ; H)\right) \rightarrow L_{\mathcal{F}_{T}}^{1}(\Omega ; H) \quad(\text { when } H \text { is a Hilbert space }), \\
\mathbb{I}(\zeta(\cdot))=\int_{0}^{T} \zeta(t) d W(t), \quad \forall \zeta(\cdot) \in L_{\mathbb{F}}^{1}\left(\Omega ; L^{2}(0, T ; H)\right)
\end{array}\right.
$$

and

$$
\left\{\begin{array}{l}
\mathbb{L}: L_{\mathbb{F}}^{1}(0, T ; H) \rightarrow L_{\mathcal{F}_{T}}^{1}(\Omega ; H), \\
\mathbb{L}(u(\cdot))=\int_{0}^{T} u(t) d t, \quad \forall u(\cdot) \in L_{\mathbb{F}}^{1}(0, T ; H) .
\end{array}\right.
$$

We call $\mathbb{I}$ and $\mathbb{L}$ the Itô integral operator and the Lebesgue integral operator, respectively. It is clear that

$$
\begin{cases}\mathbb{I}\left(L_{\mathbb{F}}^{p}\left(\Omega ; L^{2}(0, T ; H)\right)\right) \subseteq L_{\mathcal{F}_{T}}^{p}(\Omega ; H), & \forall p \in[1, \infty) \quad(\text { when } H \text { is a Hilbert space }), \\ \mathbb{L}\left(L_{\mathbb{F}}^{p}\left(\Omega ; L^{1}(0, T ; H)\right)\right) \subseteq L_{\mathcal{F}_{T}}^{p}(\Omega ; H), & \forall p \in[1, \infty) .\end{cases}
$$

The first inclusion in (1.3) can be refined (when $H$ is a Hilbert space). Indeed, for any $\xi \in L_{\mathcal{F}_{T}}^{p}(\Omega ; H)$ (with $\left.p \in[1, \infty)\right), \mathbb{E}\left[\xi \mid \mathcal{F}_{t}\right]$ is an $H$-valued continuous $L^{p}$-martingale. Hence, by the Martingale Representation Theorem ([11]), there exists a unique $\zeta(\cdot) \in$ $L_{\mathbb{F}}^{p}\left(\Omega ; L^{2}(0, T ; H)\right)$ (called the Malliavin derivative ([17]) of $\xi$ and sometimes denoted by $D . \xi)$ such that

$$
\mathbb{E}\left[\xi \mid \mathcal{F}_{t}\right]=\mathbb{E} \xi+\int_{0}^{t} \zeta(s) d W(s), \quad \forall t \in[0, T] .
$$


In particular, by taking $t=T$ in the above, we see that

$$
\xi=\mathbb{E} \xi+\int_{0}^{T} \zeta(s) d W(s) .
$$

Therefore, in the case that $H$ is a Hilbert space, the first inclusion in (1.3) can be refined to

$$
L_{\mathcal{F}_{T}}^{p}(\Omega ; H)=H \oplus\left[\mathbb{I}\left(L_{\mathbb{F}}^{p}\left(\Omega ; L^{2}(0, T ; H)\right)\right)\right],
$$

where " $\oplus$ " stands for direct sum. Now, for the second inclusion in (1.3), we have the following simple result.

Proposition 1.1. Let $H$ be a Hilbert space and $p \in[1, \infty)$. Then

$$
\overline{\mathbb{L}\left(L_{\mathbb{F}}^{p}\left(\Omega ; L^{1}(0, T ; H)\right)\right)} L_{\mathcal{F}_{T}}^{p}(\Omega ; H)=L_{\mathcal{F}_{T}}^{p}(\Omega ; H),
$$

where $\bar{G} L_{\mathcal{F}_{T}}^{p}(\Omega ; H)$ stands for the closure of $G$ in $L_{\mathcal{F}_{T}}^{p}(\Omega ; H)$.

Proof. For any $\zeta \in L_{\mathcal{F}_{T}}^{p}(\Omega ; H)$, let

$$
\xi(t)=\mathbb{E}\left[\zeta \mid \mathcal{F}_{t}\right], \quad t \in[0, T] .
$$

Then $\xi(\cdot)$ is an $H$-valued $L^{p}$-martingale. By the Martingale Representation Theorem and the Burkholder-Davis-Gundy inequality, we have

$$
\mathbb{E}|\xi(t)-\zeta|_{H}^{p} \leq C \mathbb{E}\left(\int_{t}^{T}\left|D_{s} \zeta\right|^{2} d s\right)^{p / 2} \rightarrow 0 \quad \text { as } t \rightarrow T
$$

Now, for any $\delta>0$, let

$$
u_{\delta}(t)=\frac{\xi(T-\delta)}{\delta} I_{[T-\delta, T]}(t), \quad t \in[0, T] .
$$

Then $u_{\delta}(\cdot) \in L_{\mathbb{F}}^{p}\left(\Omega ; L^{\infty}(0, T ; H)\right) \cap L_{\mathbb{F}}^{\infty}\left(0, T ; L^{p}(\Omega ; H)\right) \subseteq L_{\mathbb{F}}^{p}\left(\Omega ; L^{1}(0, T ; H)\right)$, and

$$
\mathbb{E}\left|\int_{0}^{T} u_{\delta}(t) d t-\zeta\right|^{p}=\mathbb{E}|\xi(T-\delta)-\zeta|^{p} \rightarrow 0 \quad \text { as } \delta \rightarrow 0
$$

proving the proposition.

Remark 1.2. From the proof of Proposition 1.1, it is easy to see that we have proved the following result stronger than (1.5):

${\overline{\mathbb{L}\left(L_{\mathbb{F}}^{p}\left(\Omega ; L^{\infty}(0, T ; H)\right)\right)}}^{L_{\mathcal{F}_{T}}^{p}(\Omega ; H)}={\overline{\mathbb{L}\left(L_{\mathbb{F}}^{\infty}\left(0, T ; L^{p}(\Omega ; H)\right)\right)}}_{L_{\mathcal{F}_{T}}^{p}(\Omega ; H)}=L_{\mathcal{F}_{T}}^{p}(\Omega ; H)$.

From Proposition 1.1, it is seen that we do not expect to have a refinement for the Lebesgue integral operator $\mathbb{L}$ similar to (1.4). Instead, it is very natural for us to pose the following problem: 
Problem (E). Is the following true:

$$
\mathbb{L}\left(L_{\mathbb{F}}^{p}\left(\Omega ; L^{1}(0, T ; H)\right)\right)=L_{\mathcal{F}_{T}}^{p}(\Omega ; H) ?
$$

Note that the above is equivalent to the following: When is the range of the operator $\mathbb{L}: L_{\mathbb{F}}^{p}\left(\Omega ; L^{1}(0, T ; H)\right) \rightarrow L_{\mathcal{F}_{T}}^{p}(\Omega ; H)$ closed? An interesting problem closely related to the above, taking into account (1.4), reads as follows.

Problem (R). Under what additional conditions on $\zeta(\cdot) \in L_{\mathbb{F}}^{p}\left(\Omega ; L^{2}(0, T ; H)\right)$, is there a $u(\cdot) \in L_{\mathbb{F}}^{p}\left(\Omega ; L^{1}(0, T ; H)\right)$ such that

$$
\int_{0}^{T} \zeta(t) d W(t)=\int_{0}^{T} u(t) d t \quad \text { a.s. ? }
$$

For convenience, any $u(\cdot) \in L_{\mathbb{F}}^{p}\left(\Omega ; L^{1}(0, T ; H)\right)$ satisfying $(1.7)$ is called a representor of $\zeta(\cdot)$. Since the Itô integral in the usual sense can only be defined on Hilbert spaces, we pose Problem (R) for the case that $H$ is a Hilbert space. It is clear that when $u(\cdot)$ is a representor of $\zeta(\cdot)$, so is $u(\cdot)+v(\cdot)$ as long as $\int_{0}^{T} v(t) d t=0$, almost surely. Therefore, if $\zeta(\cdot)$ admits one representor, it admits infinitely many representors. Problem (R) with $H=\mathbb{R}$ was posed and studied in [22]. Various integrability conditions were imposed on $\zeta(\cdot)$ so that it admits a representor. Let us now briefly recall several relevant results from [22], which will give us some feeling about the representation (1.7). To this end, we define

$$
u_{\alpha}(s) \equiv \frac{1-\alpha}{(T-s)^{\alpha}} \int_{0}^{s} \frac{\zeta(t)}{(T-t)^{1-\alpha}} d W(t), \quad s \in[0, T),
$$

for $\alpha \in[0,1)$. The following is a summary of the relevant results presented in [22].

Theorem 1.3. (i) Let $p \geq 1$. For any $\zeta(\cdot) \in L_{\mathbb{F}}^{p}\left(\Omega ; L^{2}(0, T ; \mathbb{R})\right)$,

$$
u_{0}(\cdot) \equiv \int_{0}^{\cdot} \frac{\zeta(t)}{T-t} d W(t) \in \bigcup_{\varepsilon>0} L_{\mathbb{F}}^{p}\left(\Omega ; L^{2}(0, T-\varepsilon ; \mathbb{R})\right),
$$

and (1.7) holds with $u(\cdot)=u_{0}(\cdot)$ in the following sense:

$$
\lim _{\varepsilon \rightarrow 0} \mathbb{E}\left|\int_{0}^{T-\varepsilon} u_{0}(t) d t-\int_{0}^{T} \zeta(t) d W(t)\right|^{p}=0 .
$$

(ii) Suppose $\zeta(\cdot) \in L_{\mathbb{F}}^{1}\left(0, T ; L^{2}(\Omega ; \mathbb{R})\right)$ is such that

$$
\int_{0}^{T}\left[\int_{0}^{s} \frac{\mathbb{E}|\zeta(t)|^{2}}{(T-t)^{2}} d t\right]^{1 / 2} d s<\infty
$$

Then

$$
u_{0}(\cdot) \equiv \int_{0}^{\cdot} \frac{\zeta(t)}{T-t} d W(t) \in L_{\mathbb{F}}^{1}(0, T ; \mathbb{R})
$$

and (1.7) holds with $u(\cdot)=u_{0}(\cdot)$. 
(iii) Suppose $\zeta(\cdot) \in L_{\mathbb{F}}^{1}(0, T ; \mathbb{R})$ is such that for some $\delta>0$,

$$
\int_{0}^{T} \frac{\mathbb{E}|\zeta(t)|^{2}}{(T-t)^{\delta}} d t<\infty
$$

Then

$u_{\alpha}(\cdot) \in L_{\mathbb{F}}^{2}\left(\Omega ; L^{q}(0, T ; \mathbb{R})\right), \quad \forall \alpha \in\left(\frac{1-\delta}{2}, \frac{1}{q}\right) \cap[0,1], q \in\left[1, \frac{2}{2-\min (\delta, 1)}\right)$,

and

$u_{\alpha}(\cdot) \in L_{\mathbb{F}}^{q}(0, T ; \mathbb{R}), \quad \forall \alpha \in\left(1-\frac{\delta}{2}-\frac{1}{q}, \frac{1}{q}\right) \cap[0,1], q \in\left[1, \frac{2}{2-\min (\delta, 1)}\right)$.

Moreover, (1.7) holds with $u(\cdot)=u_{\alpha}(\cdot)$.

(iv) Suppose $\zeta(\cdot) \in L_{\mathbb{F}}^{p}(0, T ; \mathbb{R})$ for some $p>2$. Then

$$
u_{\alpha}(\cdot) \in L_{\mathbb{F}}^{p}\left(\Omega ; L^{q}(0, T ; \mathbb{R})\right), \quad \forall \alpha \in\left(\frac{1}{2}, \frac{1}{2}+\frac{1}{p}\right) \cap[0,1], q \in\left[1, \frac{2 p}{p+2}\right) .
$$

Moreover, (1.7) holds with $u(\cdot)=u_{\alpha}(\cdot)$.

The above shows that there are many $\zeta(\cdot) \in L_{\mathbb{F}}^{p}\left(\Omega ; L^{2}(0, T ; \mathbb{R})\right)$ such that one can find a corresponding representor $u(\cdot)$.

Note that although Problem (R) is posed for the case where $H$ is a Hilbert space, Problem (E) can be posed for a general Banach space since Itô's integral is not involved here. The main purpose of this paper is to give a positive answer to Problem (E) when $H$ is a Banach space with $H^{*}$ having the Radon-Nikodým property. Our result seems to be a little surprising in some sense, and it refines the results of [22] on Problem (R). More precisely, when the answer to Problem (E) is positive, any $\zeta(\cdot) \in L_{\mathbb{F}}^{p}\left(\Omega ; L^{2}(0, T ; H)\right)$ (when $H$ is a Hilbert space) admits a representor $u(\cdot) \in L_{\mathbb{F}}^{p}\left(\Omega ; L^{1}(0, T ; H)\right)$, without assuming further integrability conditions on $\zeta(\cdot)$. This means that an Itô integral on a given (fixed) interval can be represented by a (parameterized) Bochner integral on that interval. We should emphasize here that any representor $u(\cdot)$ of $\zeta(\cdot) \in L_{\mathbb{F}}^{p}\left(\Omega ; L^{2}(0, T ; H)\right)$ depends on $T$, in general. In other words, it will be more appropriate to write

$$
\int_{0}^{T} \zeta(t) d W(t)=\int_{0}^{T} u(t, T) d t \quad \text { a.s. }
$$

Hence, by allowing the upper limit to change, we should have

$$
\int_{0}^{s} \zeta(t) d W(t)=\int_{0}^{s} u(t, s) d t, \quad \forall s \in[0, T], \quad \text { a.s. }
$$

According to Theorem 1.3, when $\zeta(\cdot)$ satisfies certain (better) integrability conditions, we can find a representor of the form

$$
u(t, s)=\frac{1-\alpha}{(s-t)^{\alpha}} \int_{0}^{t} \frac{\zeta(r)}{(s-r)^{1-\alpha}} d W(r), \quad 0 \leq t<s \leq T,
$$


for some $\alpha \in[0,1)$. Clearly, such an $s \mapsto u(t, s)$ is smooth in $s \in(t, T]$. Therefore it is natural to further ask the following question, without assuming the better integrability conditions on $\zeta(\cdot)$.

Problem (C). Does any $\zeta(\cdot) \in L_{\mathbb{F}}^{p}\left(\Omega ; L^{2}(0, T ; H)\right)$ have a representor $u(t, s)$ which is continuous with respect to the variable $s$ ?

We will also show that the answer to Problem (C) is positive. Note that, since the Itô integral $s \mapsto \int_{0}^{s} \zeta(t) d W(t)$ is at most Hölder continuous up to order $1 / 2$, generally, one cannot expect the differentiability of $s \mapsto u(t, s)$ (given in (1.9)). Nevertheless, it is natural to expect that $s \mapsto u(t, s)$ is Hölder continuous up to order $1 / 2$. But we do not have a proof for this yet.

Remark 1.4. The fact that $u(\cdot)$ in (1.8) depends on $T$ tells us that the positive answer to Problem (E) does not mean that Itô integrals can be completely replaced by (parameterized) Bochner integrals.

The rest of this paper is organized as follows. In Section 2, as a preliminary result, we establish a Riesz-type Representation Theorem for the dual of the Banach space $L_{\mathcal{M}}^{p}\left(X_{1} ; L^{q}\left(X_{2} ; H\right)\right.$ ) (see Subsection 2.1 for its definition). An interesting byproduct in this section is a characterization of the dual space of both $L_{\mathbb{F}}^{p}\left(\Omega ; L^{q}(0, T ; H)\right)$ and $L_{\mathbb{F}}^{q}\left(0, T ; L^{p}(\Omega ; H)\right)$, which will be useful in some problems in stochastic distributed parameter control systems and/or stochastic partial differential equations. Section 3 addresses Problems (E) and (R). Section 4 is devoted to answering Problem (C), for which the key tool is the continuous selection theorem of [15]. In Section 5, we present two remarkable consequences of our positive solution to Problem (E), one of which is related to a reasonable formulation of exact controllability for stochastic differential equations, and the other is a condition guaranteeing that a Black-Scholes market is complete.

\section{The dual of $L_{\mathcal{M}}^{p}\left(X_{1} ; L^{q}\left(X_{2} ; H\right)\right)$}

As a key preliminary to answering Problem $(\mathrm{E})$, we need to characterize the dual space of $L_{\mathbb{F}}^{p}\left(\Omega ; L^{q}(0, T ; H)\right)$ and $L_{\mathbb{F}}^{q}\left(0, T ; L^{p}(\Omega ; H)\right)$. We will go a little further by considering the dual of $L_{\mathcal{M}}^{p}\left(X_{1} ; L^{q}\left(X_{2} ; H\right)\right)$, to be defined below. It seems to us that this result is of independent interest.

\subsection{Statement of the result}

Let $\left(X_{1}, \mathcal{M}_{1}, \mu_{1}\right)$ and $\left(X_{2}, \mathcal{M}_{2}, \mu_{2}\right)$ be finite measure spaces. Let $\mathcal{M}$ be a sub- $\sigma$-field of $\mathcal{M}_{1} \otimes \mathcal{M}_{2}$ (the $\sigma$-field generated by $\mathcal{M}_{1} \times \mathcal{M}_{2}$ ), and for any $1 \leq p, q<\infty$, let

$$
\begin{aligned}
L_{\mathcal{M}}^{p}\left(X_{1} ; L^{q}\left(X_{2} ; H\right)\right)=\left\{\varphi: X_{1} \times X_{2} \rightarrow H\right. & H \mid \varphi(\cdot) \text { is } \mathcal{M} \text {-measurable and } \\
& \left.\int_{X_{1}}\left(\int_{X_{2}}\left|\varphi\left(x_{1}, x_{2}\right)\right|_{H}^{q} d \mu_{2}\right)^{p / q} d \mu_{1}<\infty\right\} .
\end{aligned}
$$


Likewise, let

$$
\begin{aligned}
& L_{\mathcal{M}}^{\infty}\left(X_{1} ; L^{q}\left(X_{2} ; H\right)\right)=\left\{\varphi: X_{1} \times X_{2} \rightarrow H \mid \varphi(\cdot) \text { is } \mathcal{M}\right. \text {-measurable and } \\
& \left.\underset{x_{1} \in X_{1}}{\operatorname{ess} \sup }\left(\int_{X_{2}}\left|\varphi\left(x_{1}, x_{2}\right)\right|_{H}^{q} d \mu_{2}\right)^{1 / q}<\infty\right\}, \\
& L_{\mathcal{M}}^{p}\left(X_{1} ; L^{\infty}\left(X_{2} ; H\right)\right)=\left\{\varphi: X_{1} \times X_{2} \rightarrow H \mid \varphi(\cdot) \text { is } \mathcal{M}\right. \text {-measurable and } \\
& \left.\int_{X_{1} \quad x_{2} \in X_{2}}^{\operatorname{ess} \sup }\left|\varphi\left(x_{1}, x_{2}\right)\right|_{H}^{p} d \mu_{1}<\infty\right\},
\end{aligned}
$$

$L_{\mathcal{M}}^{\infty}\left(X_{1} ; L^{\infty}\left(X_{2} ; H\right)\right)=\left\{\varphi: X_{1} \times X_{2} \rightarrow H \mid \varphi(\cdot)\right.$ is $\mathcal{M}$-measurable and

$$
\left.\underset{\left(x_{1}, x_{2}\right) \in X_{1} \times X_{2}}{\operatorname{ess} \sup _{2}}\left|\varphi\left(x_{1}, x_{2}\right)\right|_{H}<\infty\right\} .
$$

We denote

$$
L_{\mathcal{M}}^{p}\left(X_{1} \times X_{2} ; H\right)=L_{\mathcal{M}}^{p}\left(X_{1} ; L^{p}\left(X_{2} ; H\right)\right), \quad 1 \leq p \leq \infty .
$$

Also, for any $f \in L_{\mathcal{M}}^{p}\left(X_{1} ; L^{q}\left(X_{2} ; H\right)\right)(1 \leq p, q \leq \infty)$, we denote

$$
\|f\|_{p, q ; H} \equiv\|f\|_{L_{\mathcal{M}}^{p}\left(X_{1} ; L^{q}\left(X_{2} ; H\right)\right)} \triangleq\left[\int_{X_{1}}\left(\int_{X_{2}}\left|f\left(x_{1}, x_{2}\right)\right|_{H}^{q} d \mu_{2}\right)^{p / q} d \mu_{1}\right]^{1 / p} .
$$

The definitions of $\|f\|_{\infty, q, H},\|f\|_{p, \infty, H}$ and $\|f\|_{\infty, \infty, H}$ are obvious. Let

$$
\|f\|_{p ; H} \equiv\|f\|_{p, p ; H}, \quad 1 \leq p \leq \infty .
$$

The definition of $L_{\mathcal{M}}^{q}\left(X_{2} ; L^{p}\left(X_{1} ; H\right)\right)(1 \leq p, q \leq \infty)$ is similar. By Hölder's inequality and Minkowski's inequality, we have the following inclusions:

$$
L_{\mathcal{M}}^{p}\left(X_{1} ; L^{q}\left(X_{2} ; H\right)\right) \subseteq L_{\mathcal{M}}^{r}\left(X_{1} ; L^{s}\left(X_{2} ; H\right)\right), \quad 1 \leq r \leq p \leq \infty, \quad 1 \leq s \leq q \leq \infty,
$$

and (compare with (1.1)-(1.2))

$$
\begin{cases}L_{\mathcal{M}}^{p}\left(X_{1} ; L^{q}\left(X_{2} ; H\right)\right) \subseteq L_{\mathcal{M}}^{q}\left(X_{2} ; L^{p}\left(X_{1} ; H\right)\right), & 1 \leq p \leq q \leq \infty, \\ L_{\mathcal{M}}^{p}\left(X_{1} ; L^{q}\left(X_{2} ; H\right)\right) \supseteq L_{\mathcal{M}}^{q}\left(X_{2} ; L^{p}\left(X_{1} ; H\right)\right), & 1 \leq q \leq p \leq \infty .\end{cases}
$$

Next, for any $p \in[1, \infty]$, denote

$$
p^{\prime}= \begin{cases}p /(p-1), & 1<p<\infty, \\ 1, & p=\infty, \\ \infty, & p=1 .\end{cases}
$$

The definition of $q^{\prime} \in[1, \infty]$ for $q \in[1, \infty]$ is similar. 
Let $(X, \Sigma, \mu)$ be a finite measure space and $B$ a Banach space. We recall that $B$ is said to have the Radon-Nikodým property with respect to $(X, \Sigma, \mu)$ if, for every countably additive vector measure $v$ on $(X, \Sigma)$ with values in $B$ which has bounded variation and is absolutely continuous with respect to $\mu$, there is a $\mu$-(Bochner) integrable function $g: X \rightarrow B$ such that

$$
\nu(E)=\int_{E} g d \mu, \quad \forall E \in \Sigma .
$$

The Banach space $B$ is said to have the Radon-Nikodým property if $B$ has the RadonNikodým property with respect to every finite measure space. It is known that spaces with the Radon-Nikodým property include reflexive spaces, in particular, Hilbert spaces (e.g. [7]).

We have the following result.

Lemma 2.1. Let $H$ be a Banach space, $\left(X_{1}, \mathcal{M}_{1}, \mu_{1}\right)$ and $\left(X_{2}, \mathcal{M}_{2}, \mu_{2}\right)$ be finite measure spaces, $\mathcal{M}$ be a sub- $\sigma$-field of $\mathcal{M}_{1} \otimes \mathcal{M}_{2}$, and let $1 \leq p, q<\infty$. Then $H^{*}$ has the Radon-Nikodým property with respect to $\left(X_{1} \times X_{2}, \mathcal{M}, \mu_{1} \times \mu_{2}\right)$ if and only if for any $F \in L_{\mathcal{M}}^{p}\left(X_{1} ; L^{q}\left(X_{2} ; H\right)\right)^{*}$, there exists a unique $g \in L_{\mathcal{M}}^{p^{\prime}}\left(X_{1} ; L^{q^{\prime}}\left(X_{2} ; H^{*}\right)\right)$ such that

$$
F(f)=\int_{X_{1} \times X_{2}}\left(f\left(x_{1}, x_{2}\right), g\left(x_{1}, x_{2}\right)\right)_{H, H^{*}} d \mu_{1} d \mu_{2}, \quad \forall f \in L_{\mathcal{M}}^{p}\left(X_{1} ; L^{q}\left(X_{2} ; H\right)\right),
$$

and

$$
\|F\|_{L_{\mathcal{M}}^{p}\left(X_{1} ; L^{q}\left(X_{2} ; H\right)\right)^{*}}=\|g\|_{p^{\prime}, q^{\prime}, H^{*}}
$$

Due to the above result, we make the following identification (for the case that $H^{*}$ has the Radon-Nikodým property with respect to $\left.\left(X_{1} \times X_{2}, \mathcal{M}, \mu_{1} \times \mu_{2}\right)\right)$ :

$$
L_{\mathcal{M}}^{p}\left(X_{1} ; L^{q}\left(X_{2} ; H\right)\right)^{*}=L_{\mathcal{M}}^{p^{\prime}}\left(X_{1} ; L^{q^{\prime}}\left(X_{2} ; H^{*}\right)\right), \quad 1 \leq p, q<\infty
$$

The above is a Riesz-type Representation Theorem for the dual of $L_{\mathcal{M}}^{p}\left(X_{1} ; L^{q}\left(X_{2} ; H\right)\right)$. It seems to us that Lemma 2.1 should be a known result but we have not found an exact reference. Therefore, for the reader's convenience, we provide a detailed proof in the next three subsections. As a corollary of Lemma 2.1, we will characterize the dual of $L_{\mathbb{F}}^{p}\left(\Omega ; L^{q}(0, T ; H)\right)$ and $L_{\mathbb{F}}^{q}\left(0, T ; L^{p}(\Omega ; H)\right)$ in the last subsection.

The main idea for the proof of Lemma 2.1 is similar to that of the relevant result in [4, Appendix B, pp. 375-376] (see also [7, Theorem 1, Chapter IV, pp. 98-99]). However, Lemma 2.1 does not follow from the main result in [4, Appendix B] because the latter considered only the special case that $p=q$ and $H=\mathbb{R}$, for which, by Fubini's Theorem, one can reduce the problem to the case with one measure on the product space. Also, Lemma 2.1 does not seem to be a corollary of [7, Theorem 1, Chapter IV, pp. 98-99] because of the very fact that our $\mathcal{M}$ is an "interconnecting" sub- $\sigma$-field of the $\sigma$-field generated by $\mathcal{M}_{1} \times \mathcal{M}_{2}$. 


\subsection{Proof of the necessity in Lemma 2.1 for $H=\mathbb{R}$}

As a key step in proving Lemma 2.1, in this subsection we show first the "only if" part of this lemma for the special case $H=\mathbb{R}$.

For any $g \in L_{\mathcal{M}}^{p^{\prime}}\left(X_{1} ; L^{q^{\prime}}\left(X_{2} ; \mathbb{R}\right)\right)$, define $F_{g}: L_{\mathcal{M}}^{p}\left(X_{1} ; L^{q}\left(X_{2} ; \mathbb{R}\right)\right) \rightarrow \mathbb{R}$ by

$$
F_{g}(f)=\int_{X_{1} \times X_{2}} f\left(x_{1}, x_{2}\right) g\left(x_{1}, x_{2}\right) d \mu_{1} d \mu_{2}, \quad \forall f \in L_{\mathcal{M}}^{p}\left(X_{1} ; L^{q}\left(X_{2} ; \mathbb{R}\right)\right) .
$$

By the linearity of the integral, $g \mapsto F_{g}$ is a linear map. It follows from Hölder's inequality that

$$
\left|F_{g}(f)\right| \leq\|f\|_{p, q, \mathbb{R}}\|g\|_{p^{\prime}, q^{\prime}, \mathbb{R}}, \quad \forall f \in L_{\mathcal{M}}^{p}\left(X_{1} ; L^{q}\left(X_{2} ; \mathbb{R}\right)\right) .
$$

Hence $F_{g} \in L_{\mathcal{M}}^{p}\left(X_{1} ; L^{q}\left(X_{2} ; \mathbb{R}\right)\right)^{*}$ and

$$
\left\|F_{g}\right\|_{L_{\mathcal{M}}^{p}\left(X_{1} ; L^{q}\left(X_{2} ; \mathbb{R}\right)\right)^{*}} \leq\|g\|_{p^{\prime}, q^{\prime}, \mathbb{R}} .
$$

Therefore, $g \mapsto F_{g}$ is a linear nonexpanding map. Now, we show that this map is surjective and is an isometry.

To show the surjectivity of $g \mapsto F_{g}$, take any $F \in L_{\mathcal{M}}^{p}\left(X_{1} ; L^{q}\left(X_{2} ; \mathbb{R}\right)\right)^{*}$. Since for any $A \in \mathcal{M}, I_{A} \in L_{\mathcal{M}}^{p}\left(X_{1} ; L^{q}\left(X_{2} ; \mathbb{R}\right)\right)$, we may define

$$
v(A)=F\left(I_{A}\right), \quad \forall A \in \mathcal{M} .
$$

Then $v$ is a totally finite signed measure on $\left(X_{1} \times X_{2}, \mathcal{M}\right)$, and $v<<\mu_{1} \times \mu_{2}$. By the Radon-Nikodým Theorem, there is an $\mathcal{M}$-measurable map $g \in L_{\mathcal{M}}^{1}\left(X_{1} \times X_{2} ; \mathbb{R}\right)$ such that

$$
v(A)=\int_{A} g d \mu_{1} d \mu_{2}, \quad \forall A \in \mathcal{M},
$$

i.e.,

$$
F\left(I_{A}\right)=\int_{X_{1} \times X_{2}} g I_{A} d \mu_{1} d \mu_{2}, \quad \forall A \in \mathcal{M} .
$$

Consequently, for any $\mathcal{M}$-measurable simple function $f$,

$$
F(f)=\int_{X_{1} \times X_{2}} f\left(x_{1}, x_{2}\right) g\left(x_{1}, x_{2}\right) d \mu_{1} d \mu_{2} .
$$

Select a sequence $\left\{A_{n}\right\}_{n=1}^{\infty} \subset \mathcal{M}$ such that

$$
A_{n} \subset A_{n+1}, \quad n=1,2, \ldots, \quad\left(\mu_{1} \times \mu_{2}\right)\left(\left(X_{1} \times X_{2}\right) \backslash \bigcup_{n=1}^{\infty} A_{n}\right)=0,
$$

and $g$ is bounded on each $A_{n}$. For any $n \geq 1$, note that

$$
f \mapsto \int_{X_{1} \times X_{2}} f\left(x_{1}, x_{2}\right) g\left(x_{1}, x_{2}\right) I_{A_{n}}\left(x_{1}, x_{2}\right) d \mu_{1} d \mu_{2}
$$


is a bounded linear functional on $L_{\mathcal{M}}^{p}\left(X_{1} ; L^{q}\left(X_{2} ; \mathbb{R}\right)\right)$ which agrees with $F$ on all $\mathcal{M}$ measurable simple functions which vanish off $A_{n}$. It follows that

$$
F\left(f I_{A_{n}}\right)=\int_{X_{1} \times X_{2}} f g I_{A_{n}} d \mu_{1} d \mu_{2}, \quad \forall f \in L_{\mathcal{M}}^{p}\left(X_{1} ; L^{q}\left(X_{2} ; \mathbb{R}\right)\right) .
$$

Since $g I_{A_{n}}$ is bounded, one sees that $g I_{A_{n}} \in L_{\mathcal{M}}^{p^{\prime}}\left(X_{1} ; L^{q^{\prime}}\left(X_{2} ; \mathbb{R}\right)\right)$. We claim that $g \in$ $L_{\mathcal{M}}^{p^{\prime}}\left(X_{1} ; L^{q^{\prime}}\left(X_{2} ; \mathbb{R}\right)\right)$, and

$$
\|g\|_{p^{\prime}, q^{\prime} ; \mathbb{R}} \leq\|F\|_{L_{\mathcal{M}}^{p}\left(X_{1} ; L^{q}\left(X_{2} ; \mathbb{R}\right)\right)^{*}}
$$

To show this, we distinguish four cases.

Case 1: $p, q \in(1, \infty)$. Choose

$$
f= \begin{cases}a\left(\int_{X_{2}}|g|^{q^{\prime}} I_{A_{n}} d \mu_{2}\right)^{p^{\prime} / q^{\prime}-1}|g|^{q^{\prime}-1}(\operatorname{sgn} g) I_{A_{n}} & \text { if } \int_{X_{2}}|g|^{q^{\prime}} I_{A_{n}} d \mu_{2} \neq 0, \\ 0, & \text { if } \int_{X_{2}}|g|^{q^{\prime}} I_{A_{n}} d \mu_{2}=0,\end{cases}
$$

where

$$
a=\left[\int_{X_{1}}\left(\int_{X_{2}}|g|^{q^{\prime}} I_{A_{n}} d \mu_{2}\right)^{p^{\prime} / q^{\prime}} d \mu_{1}\right]^{1 / p^{\prime}-1} .
$$

Then

$$
\begin{aligned}
\|f\|_{p, q} & =\left[\int_{X_{1}}\left(\int_{X_{2}}|f|^{q} d \mu_{2}\right)^{p / q} d \mu_{1}\right]^{1 / p} \\
& =\left\{\int_{X_{1}}\left[\int_{X_{2}} a^{q}\left(\int_{X_{2}}|g|^{q^{\prime}} I_{A_{n}} d \mu_{2}\right)^{\left(p^{\prime} / q^{\prime}-1\right) q}|g|^{\left(q^{\prime}-1\right) q} I_{A_{n}} d \mu_{2}\right]^{p / q} d \mu_{1}\right\}^{1 / p} \\
& =a\left\{\int_{X_{1}}\left[\left(\int_{X_{2}}|g|^{q^{\prime}} I_{A_{n}} d \mu_{2}\right)^{\left(p^{\prime} / q^{\prime}-1\right) p}\left(\int_{X_{2}}|g|^{q^{\prime}} I_{A_{n}} d \mu_{2}\right)^{p / q}\right] d \mu_{1}\right\}^{1 / p} \\
& =a\left\{\int_{X_{1}}\left(\int_{X_{2}}|g|^{q^{\prime}} I_{A_{n}} d \mu_{2}\right)^{p^{\prime} / q^{\prime}} d \mu_{1}\right\}^{1 / p}=1 .
\end{aligned}
$$

Taking the above $f$ in (2.4), we find that

$$
\begin{aligned}
F(f) & =\int_{X_{1}} \int_{X_{2}} f g I_{A_{n}} d \mu_{2} d \mu_{1} \\
& =a \int_{X_{1}}\left[\int_{X_{2}}\left(\int_{X_{2}}|g|^{q^{\prime}} I_{A_{n}} d \mu_{2}\right)^{p^{\prime} / q^{\prime}-1}|g|^{q^{\prime}} I_{A_{n}} d \mu_{2}\right] d \mu_{1} \\
& =a \int_{X_{1}}\left(\int_{X_{2}}|g|^{q^{\prime}} I_{A_{n}} d \mu_{2}\right)^{p^{\prime} / q^{\prime}} d \mu_{1}=\left[\int_{X_{1}}\left(\int_{X_{2}}|g|^{q^{\prime}} I_{A_{n}} d \mu_{2}\right)^{p^{\prime} / q^{\prime}} d \mu_{1}\right]^{1 / p^{\prime}} \\
& =\left\|g I_{A_{n}}\right\|_{p^{\prime}, q^{\prime} ; \mathbb{R}},
\end{aligned}
$$


which gives

$$
\left\|g I_{A_{n}}\right\|_{p^{\prime}, q^{\prime} ; \mathbb{R}} \leq\|F\|_{L_{\mathcal{M}}^{p}\left(X_{1} ; L^{q}\left(X_{2} ; \mathbb{R}\right)\right)^{*}}
$$

Letting $n \rightarrow \infty$, by making use of Fatou's Lemma, one deduces (2.5).

Case 2: $p=1,1<q<\infty$. In this case, we first take $p \in(1, \infty)$, and take $f$ as in Case 1. Then

$$
\begin{aligned}
\|f\|_{1, q} & =\int_{X_{1}}\left(\int_{X_{2}}|f|^{q} d \mu_{2}\right)^{1 / q} d \mu_{1} \leq\left[\int_{X_{1}}\left(\int_{X_{2}}|f|^{q} d \mu_{2}\right)^{p / q} d \mu_{1}\right]^{1 / p} \mu_{1}\left(X_{1}\right)^{1 / p^{\prime}} \\
& =\mu_{1}\left(X_{1}\right)^{1 / p^{\prime}} .
\end{aligned}
$$

Consequently,

$$
\begin{aligned}
\left\|g I_{A_{n}}\right\|_{p^{\prime}, q^{\prime} ; \mathbb{R}} & =F(f) \leq\|F\|_{L_{\mathcal{M}}^{1}\left(X_{1} ; L^{q}\left(X_{2} ; \mathbb{R}\right)\right)^{*}}\|f\|_{1, q} \\
& \leq\|F\|_{L_{\mathcal{M}}^{1}\left(X_{1} ; L^{q}\left(X_{2} ; \mathbb{R}\right)\right)^{*}} \mu_{1}\left(X_{1}\right)^{1 / p^{\prime}} .
\end{aligned}
$$

Letting $n \rightarrow \infty$ and then $p \rightarrow 1$ (which means $p^{\prime} \rightarrow \infty$ ), we obtain

$$
\|g\|_{\infty, q^{\prime} ; \mathbb{R}} \leq\|F\|_{L_{\mathcal{M}}^{1}\left(X_{1} ; L^{q}\left(X_{2} ; \mathbb{R}\right)\right)^{*}}
$$

which is (2.5) for $p=1$.

Case 3: $1<p<\infty, q=1$. In this case, we first take $q \in(1, \infty)$, and take $f$ as in Case 1. Then

$$
\begin{aligned}
\|f\|_{p, 1 ; \mathbb{R}} & =\left[\int_{X_{1}}\left(\int_{X_{2}}|f| d \mu_{2}\right)^{p} d \mu_{1}\right]^{1 / p} \\
& \leq\left[\int_{X_{1}}\left(\int_{X_{2}}|f|^{q} d \mu_{2}\right)^{p / q} d \mu_{1}\right]^{1 / p} \mu_{2}\left(X_{2}\right)^{1 / q^{\prime}}=\mu_{2}\left(X_{2}\right)^{1 / q^{\prime}} .
\end{aligned}
$$

Hence,

$$
\begin{aligned}
\left\|g I_{A_{n}}\right\|_{p^{\prime}, q^{\prime} ; \mathbb{R}} & =F(f) \leq\|F\|_{L_{\mathcal{M}}^{p}\left(X_{1} ; L^{1}\left(X_{2} ; \mathbb{R}\right)\right)^{*}}\|f\|_{p, 1 ; \mathbb{R}} \\
& \leq\|F\|_{L_{\mathcal{M}}^{p}\left(X_{1} ; L^{1}\left(X_{2} ; \mathbb{R}\right)\right)^{*}} \mu_{2}\left(X_{1}\right)^{1 / q^{\prime}}
\end{aligned}
$$

Letting $n \rightarrow \infty$ and then $q \rightarrow 1$ (which means $q^{\prime} \rightarrow \infty$ ), we obtain

$$
\|g\|_{p^{\prime}, \infty ; \mathbb{R}} \leq\|F\|_{L_{\mathcal{M}}^{p}\left(X_{1} ; L^{1}\left(X_{2} ; \mathbb{R}\right)\right)^{*}},
$$

which is (2.5) for $q=1$. 
Case 4: $p=q=1$. In this case, we still first let $p, q \in(1, \infty)$, and take $f$ as in Case 1 with $q=r$. Then

$$
\begin{aligned}
\|f\|_{1,1} & =\int_{X_{1}} \int_{X_{2}}|f| d \mu_{2} d \mu_{1} \\
& \leq\left[\int_{X_{1}}\left(\int_{X_{2}}|f|^{q} d \mu_{2}\right)^{p / q} d \mu_{1}\right]^{1 / p} \mu_{1}\left(X_{1}\right)^{1 / p^{\prime}} \mu_{2}\left(X_{2}\right)^{1 / q^{\prime}} \\
& =\mu_{1}\left(X_{1}\right)^{1 / p^{\prime}} \mu_{2}\left(X_{2}\right)^{1 / q^{\prime}} .
\end{aligned}
$$

Consequently,

$$
\begin{aligned}
\left\|g I_{A_{n}}\right\|_{p^{\prime}, q^{\prime} ; \mathbb{R}} & =F(f) \leq\|F\|_{L_{\mathcal{M}}^{1}\left(X_{1} ; L^{1}\left(X_{2} ; \mathbb{R}\right)\right)^{*}}\|f\|_{1,1} \\
& \leq\|F\|_{L_{\mathcal{M}}^{1}\left(X_{1} ; L^{1}\left(X_{2} ; \mathbb{R}\right)\right)^{*}} \mu_{1}\left(X_{1}\right)^{1 / p^{\prime}} \mu_{2}\left(X_{1}\right)^{1 / q^{\prime}} .
\end{aligned}
$$

Letting $n \rightarrow \infty$ and then $p, q \rightarrow 1$ (which means $p^{\prime}, q^{\prime} \rightarrow \infty$ ), we obtain

$$
\|g\|_{\infty ; \mathbb{R}} \leq\|F\|_{L_{\mathcal{M}}^{1}\left(X_{1} ; L^{1}\left(X_{2} ; \mathbb{R}\right)\right)^{*}},
$$

which is (2.5) for $p, q=1$.

Finally, (2.3) means that $F_{g} \in\left(L_{\mathcal{M}}^{p}\left(X_{1} ; L^{q}\left(X_{2} ; \mathbb{R}\right)\right)\right)^{*}$ and since $F$ and $F_{g}$ coincides on a dense subset of $L_{\mathcal{M}}^{p}\left(X_{1} ; L^{q}\left(X_{2} ; \mathbb{R}\right)\right)$, one has $F=F_{g}$. Also, (2.2) follows easily from (2.3) and (2.5).

\subsection{Proof of the necessity in Lemma 2.1 for the general case}

We are now in a position to prove the "only if" part of Lemma 2.1 for the general case. The proof is divided into two steps.

Step 1. We show that $L_{\mathcal{M}}^{p^{\prime}}\left(X_{1} ; L^{q^{\prime}}\left(X_{2} ; H^{*}\right)\right)$ is isometrically isomorphic to a subspace $\mathcal{H}$ of $L_{\mathcal{M}}^{p}\left(X_{1} ; L^{q}\left(X_{2} ; H\right)\right)^{*}$.

For any given $g \in L_{\mathcal{M}}^{p^{\prime}}\left(X_{1} ; L^{q^{\prime}}\left(X_{2} ; H^{*}\right)\right)$, define a linear functional $F_{g}$ on the space $L_{\mathcal{M}}^{p}\left(X_{1} ; L^{q}\left(X_{2} ; H\right)\right)$ as follows:

$$
F_{g}(f)=\int_{X_{1} \times X_{2}}\left\langle f\left(x_{1}, x_{2}\right), g\left(x_{1}, x_{2}\right)\right\rangle_{H, H^{*}} d \mu_{1} d \mu_{2}, \quad \forall f \in L_{\mathcal{M}}^{p}\left(X_{1} ; L^{q}\left(X_{2} ; H\right)\right) .
$$

Then, by means of the Hölder inequality and similar to (2.3), we conclude that $F_{g}$ belongs to $L_{\mathcal{M}}^{p}\left(X_{1} ; L^{q}\left(X_{2} ; H\right)\right)^{*}$, and

$$
\left\|F_{g}\right\|_{L_{\mathcal{M}}^{p}\left(X_{1} ; L^{q}\left(X_{2} ; H\right)\right)^{*}} \leq\|g\|_{p^{\prime} q^{\prime} ; H^{*}}
$$

Therefore the norm of $F_{g}$ is not greater than $\|g\|_{p^{\prime} q^{\prime}, H^{*} \text {. Define }}$

$$
\mathcal{H} \equiv\left\{F_{g} \mid g \in L_{\mathcal{M}}^{p^{\prime}}\left(X_{1} ; L^{q^{\prime}}\left(X_{2} ; H^{*}\right)\right)\right\} .
$$


It remains to prove the reverse of inequality (2.6). Clearly, without loss of generality, we may assume that $g \neq 0$.

Suppose first that $g=\sum_{i=1}^{\infty} h_{i}^{*} I_{E_{i}}$ where $h_{i}^{*}$ is a sequence in $H^{*}$ and $\left\{E_{i}\right\}_{i=1}^{\infty}$ is a countable partition of $X_{1} \times X_{2}$ by members of $\mathcal{M}$ with $\left(\mu_{1} \times \mu_{2}\right)\left(E_{i}\right)>0$ for all $i$. Since we have shown that $L_{\mathcal{M}}^{p}\left(X_{1} ; L^{q}\left(X_{2} ; \mathbb{R}\right)\right)^{*}=L_{\mathcal{M}}^{p^{\prime}}\left(X_{1} ; L^{q^{\prime}}\left(X_{2} ; \mathbb{R}\right)\right.$ ) (in Subsection 2.2) and noting that $0<|g|_{H^{*}} \in L_{\mathcal{M}^{p^{\prime}}}\left(X_{1} ; L^{q^{\prime}}\left(X_{2} ; \mathbb{R}\right)\right)$, for any $\varepsilon>0$ there exists a nonnegative function $\varphi \in L_{\mathcal{M}}^{p}\left(X_{1} ; L^{q}\left(X_{2} ; \mathbb{R}\right)\right)$ such that

$$
0<\|\varphi\|_{p, q} \leq 1, \quad\|g\|_{p^{\prime} q^{\prime} ; H^{*}}-\varepsilon \leq \int_{X_{1} \times X_{2}}|g|_{H^{*}} \varphi d \mu_{1} d \mu_{2} .
$$

Further, choose $h_{i} \in H$ with $\left|h_{i}\right|_{H}=1$ such that

$$
\left|h_{i}^{*}\right|_{H^{*}}-\varepsilon /\|\varphi\|_{1,1} \leq h_{i}^{*}\left(h_{i}\right),
$$

and define

$$
f=\sum_{i=1}^{\infty} \varphi h_{i} I_{E_{i}} \in L_{\mathcal{M}}^{p}\left(X_{1} ; L^{q}\left(X_{2} ; H\right)\right) .
$$

Then $\|f\|_{p, q, H}=\|\varphi\|_{p, q} \leq 1$, and we have

$$
\begin{aligned}
\int_{X_{1} \times X_{2}}\left\langle f\left(x_{1}, x_{2}\right), g\left(x_{1}, x_{2}\right)\right\rangle_{H, H^{*}} d \mu_{1} d \mu_{2}=\int_{X_{1} \times X_{2}} \varphi \sum_{i=1}^{\infty}\left\langle h_{i}, h_{i}^{*}\right\rangle_{H, H^{*}} I_{E_{i}} d \mu_{1} d \mu_{2} \\
\quad \geq \int_{X_{1} \times X_{2}} \varphi \sum_{i=1}^{\infty}\left(\left|h_{i}^{*}\right|_{H^{*}}-\frac{\varepsilon}{\|\varphi\|_{1,1}}\right) I_{E_{i}} d \mu_{1} d \mu_{2} \\
\geq \int_{X_{1} \times X_{2}}|g|_{H^{*}} \varphi d \mu_{1} d \mu_{2}-\frac{\varepsilon}{\|\varphi\|_{1,1}} \int_{X_{1} \times X_{2}} \varphi d \mu_{1} d \mu_{2} \geq\|g\|_{p^{\prime}, q^{\prime}, H^{*}}-2 \varepsilon
\end{aligned}
$$

This gives

$$
\left\|F_{g}\right\|_{L_{\mathcal{M}}^{p}\left(X_{1} ; L^{q}\left(X_{2} ; H\right)\right)^{*}} \geq\|g\|_{p^{\prime} q^{\prime} ; H^{*}},
$$

and therefore

$$
\left\|F_{g}\right\|_{L_{\mathcal{M}}^{p}\left(X_{1} ; L^{q}\left(X_{2} ; H\right)\right)^{*}}=\|g\|_{p^{\prime} q^{\prime} ; H^{*}},
$$

whenever $g \in L_{\mathcal{M}}^{p^{\prime}}\left(X_{1} ; L^{q^{\prime}}\left(X_{2} ; H^{*}\right)\right)$ is countably valued.

For the general case, we choose a sequence $\left\{g_{n}\right\}_{n=1}^{\infty} \subset L_{\mathcal{M}}^{p^{\prime}}\left(X_{1} ; L^{q^{\prime}}\left(X_{2} ; H^{*}\right)\right)$ such that each $g_{n}$ is countably valued and

$$
\lim _{n \rightarrow \infty}\left\|g_{n}-g\right\|_{p^{\prime}, q^{\prime} ; H^{*}}=0
$$

We have obtained

$$
\left\|F_{g_{n}}\right\|_{L_{\mathcal{M}}^{p}\left(X_{1} ; L^{q}\left(X_{2} ; H\right)\right)^{*}}=\left\|g_{n}\right\|_{p^{\prime}, q^{\prime} ; H^{*}}
$$

and by virtue of (2.6),

$$
\left\|F_{g_{n}}-F_{g}\right\|_{L_{\mathcal{M}}^{p}\left(X_{1} ; L^{q}\left(X_{2} ; H\right)\right)^{*}}=\left\|F_{g_{n}-g}\right\|_{L_{\mathcal{M}}^{p}\left(X_{1} ; L^{q}\left(X_{2} ; H\right)\right)^{*}} \leq\left\|g_{n}-g\right\|_{p^{\prime}, q^{\prime} ; H^{*}}
$$


Therefore, noting (2.7), we end up with

$$
\begin{aligned}
\left\|F_{g}\right\|_{L_{\mathcal{M}}^{p}\left(X_{1} ; L^{q}\left(X_{2} ; H\right)\right)^{*}} & =\lim _{n \rightarrow \infty}\left\|F_{g_{n}}\right\|_{L_{\mathcal{M}}^{p}\left(X_{1} ; L^{q}\left(X_{2} ; H\right)\right)^{*}} \\
& =\lim _{n \rightarrow \infty}\left\|g_{n}\right\|_{p^{\prime} q^{\prime}, H^{*}}=\|g\|_{p^{\prime} q^{\prime} ; H^{*}}
\end{aligned}
$$

Hence we conclude that $L_{\mathcal{M}}^{p^{\prime}}\left(X_{1} ; L^{q^{\prime}}\left(X_{2} ; H^{*}\right)\right)$ is isometrically isomorphic to $\mathcal{H}$.

Step 2. We show that the subspace $\mathcal{H}$ is equal to $L_{\mathcal{M}}^{p}\left(X_{1} ; L^{q}\left(X_{2} ; H\right)\right)^{*}$.

To this end, for $F \in L_{\mathcal{M}}^{p}\left(X_{1} ; L^{q}\left(X_{2} ; H\right)\right)^{*}$, we define

$$
G(E)(h)=F\left(h I_{E}\right), \quad \forall E \in \mathcal{M}, h \in H .
$$

From

$$
\left|F\left(h I_{E}\right)\right| \leq\|F\|_{L_{\mathcal{M}}^{p}\left(X_{1} ; L^{q}\left(X_{2} ; H\right)\right)^{*}}\left\|h I_{E}\right\|_{p, q, H} \leq\|F\|_{L_{\mathcal{M}}^{p}\left(X_{1} ; L^{q}\left(X_{2} ; H\right)\right)^{*}}|h|_{H}\left\|I_{E}\right\|_{p, q},
$$

we see that $G: \mathcal{M} \rightarrow H^{*}$ and it is countably additive. Let $E_{1}, \ldots, E_{n}(n \in \mathbb{N})$ be a partition of $X_{1} \times X_{2}$ by members of $\mathcal{M}$ with $\left(\mu_{1} \times \mu_{2}\right)\left(E_{i}\right)>0$ for all $1 \leq i \leq n$. Then $G\left(E_{i}\right) \in H^{*}$. Define

$$
G_{E_{i}}^{1}(h)=\operatorname{Re} G(E)(h), \quad G_{E_{i}}^{2}(h)=\operatorname{Im} G(E)(h), \quad \forall h \in H .
$$

Clearly, $\left|G\left(E_{i}\right)\right|_{H^{*}} \leq\left|G_{E_{i}}^{1}\right|_{H^{*}}+\left|G_{E_{i}}^{2}\right|_{H^{*}}$. Noting that both $G_{E_{i}}^{1}$ and $G_{E_{i}}^{2}$ are real functionals, we see that, for any $\varepsilon>0$, one can find $h_{i}^{1}$ and $h_{i}^{2}$ in the closed unit ball of $H$ such that

$$
\left|G_{E_{i}}^{1}\right|_{H^{*}}-\frac{\varepsilon}{2 n}<\operatorname{Re} G\left(E_{i}\right)\left(h_{i}^{1}\right), \quad\left|G_{E_{i}}^{2}\right|_{H^{*}}-\frac{\varepsilon}{2 n}<\operatorname{Im} G\left(E_{i}\right)\left(h_{i}^{2}\right) .
$$

It follows that

$$
\begin{aligned}
\sum_{i=1}^{n}\left|G\left(E_{i}\right)\right|_{H^{*}}-\varepsilon & <\operatorname{Re} \sum_{i=1}^{n} G\left(E_{i}\right)\left(h_{i}^{1}\right)+\operatorname{Im} \sum_{i=1}^{n} G\left(E_{i}\right)\left(h_{i}^{2}\right) \\
& =\operatorname{Re} F\left(\sum_{i=1}^{n} h_{i}^{1} I_{E_{i}}\right)+\operatorname{Im} F\left(\sum_{i=1}^{n} h_{i}^{2} I_{E_{i}}\right) \\
& \leq\|F\|_{L_{\mathcal{M}}^{p}\left(X_{1} ; L^{q}\left(X_{2} ; H\right)\right)^{*}}\left(\left\|\sum_{i=1}^{n} h_{i}^{1} I_{E_{i}}\right\|_{p, q, H}+\left\|\sum_{i=1}^{n} h_{i}^{2} I_{E_{i}}\right\|_{p, q, H}\right) \\
& \leq 2\|F\|_{L_{\mathcal{M}}^{p}\left(X_{1} ; L^{q}\left(X_{2} ; H\right)\right)^{*}}\left\|\sum_{i=1}^{n} I_{E_{i}}\right\|_{p, q} \\
& \leq 2\|F\|_{L_{\mathcal{M}}^{p}\left(X_{1} ; L^{q}\left(X_{2} ; H\right)\right)^{*}} \mu_{1}\left(X_{1}\right)^{1 / p} \mu_{2}\left(X_{2}\right)^{1 / q} .
\end{aligned}
$$

Hence $\left|G\left(X_{1} \times X_{2}\right)\right|_{H^{*}}<\infty$ and $G$ is a $\left(\mu_{1} \times \mu_{2}\right)$-continuous vector-valued measure of bounded variation. Since $H^{*}$ has the Radon-Nikodým property with respect to $\left(X_{1} \times X_{2}\right.$, $\left.\mathcal{M}, \mu_{1} \times \mu_{2}\right)$, there exists a Bochner integrable $g: X_{1} \times X_{2} \rightarrow H^{*}$ such that

$$
G(E)=\int_{E} g d \mu_{1} d \mu_{2}, \quad \forall E \in \mathcal{M}
$$


Clearly, if $f \in L_{\mathcal{M}}^{p}\left(X_{1} ; L^{q}\left(X_{2} ; H\right)\right)$ is a simple function, then

$$
F(f)=\int_{X_{1} \times X_{2}}\left\langle f\left(x_{1}, x_{2}\right), g\left(x_{1}, x_{2}\right)\right\rangle_{H, H^{*}} d \mu_{1} d \mu_{2} .
$$

Select an expanding sequence $\left\{E_{n}\right\}_{n=1}^{\infty}$ in $\mathcal{M}$ such that $\bigcup_{n=1}^{\infty} E_{n}=X_{1} \times X_{2}$ and such that $g$ is bounded on each $E_{n}$. Fixing $n_{0} \in \mathbb{N}$ and noting that the linear functional $\int_{E_{n_{0}}}\left\langle\cdot, g\left(x_{1}, x_{2}\right)\right\rangle_{H, H^{*}} d \mu_{1} d \mu_{2}$ is a bounded linear functional on $L_{\mathcal{M}}^{p}\left(X_{1} ; L^{q}\left(X_{2} ; H\right)\right)$ which agrees with $F$ on all simple functions supported on $E_{n_{0}}$, it follows that

$$
\begin{aligned}
F\left(f I_{E_{n_{0}}}\right)=\int_{X_{1} \times X_{2}}\left\langle f\left(x_{1}, x_{2}\right), g\left(x_{1}, x_{2}\right) I_{E_{n_{0}}}\right\rangle_{H, H^{*}} d \mu_{1} d \mu_{2}, & \\
& \forall f \in L_{\mathcal{M}}^{p}\left(X_{1} ; L^{q}\left(X_{2} ; H\right)\right) .
\end{aligned}
$$

Further, since $g I_{E_{n_{0}}}$ is bounded, one has $g I_{E_{n_{0}}} \in L_{\mathcal{M}}^{p^{\prime}}\left(X_{1} ; L^{q^{\prime}}\left(X_{2} ; H^{*}\right)\right)$ and

$$
\left\|g I_{E_{n_{0}}}\right\|_{p^{\prime}, q^{\prime} ; H^{*}} \leq\|F\|_{L_{\mathcal{M}}^{p}\left(X_{1} ; L^{q}\left(X_{2} ; H\right)\right)^{*}} .
$$

Since inequality (2.9) holds for each $n_{0}$, by the Monotone Convergence Theorem, we conclude that $g \in L_{\mathcal{M}}^{p^{\prime}}\left(X_{1} ; L^{q^{\prime}}\left(X_{2} ; H^{*}\right)\right)$.

Finally, for any $f \in L_{\mathcal{M}}^{p}\left(X_{1} ; L^{q}\left(X_{2} ; H\right)\right)$, it follows from (2.8) that

$$
\begin{aligned}
F(f) & =\lim _{n \rightarrow \infty} \int_{X_{1} \times X_{2}}\left\langle f\left(x_{1}, x_{2}\right), g\left(x_{1}, x_{2}\right) I_{E_{n}}\right\rangle_{H, H^{*}} d \mu_{1} d \mu_{2} \\
& =\int_{X_{1} \times X_{2}}\left\langle f\left(x_{1}, x_{2}\right), g\left(x_{1}, x_{2}\right)\right\rangle_{H, H^{*}} d \mu_{1} d \mu_{2}=F_{g}(f) .
\end{aligned}
$$

This means that $F=F_{g}$. Hence $L_{\mathcal{M}}^{p}\left(X_{1} ; L^{q}\left(X_{2} ; H\right)\right)^{*}$ coincides with the linear space $L_{\mathcal{M}}^{p^{\prime}}\left(X_{1} ; L^{q^{\prime}}\left(X_{2} ; H^{*}\right)\right)$.

\subsection{Proof of the sufficiency in Lemma 2.1}

In order to complete the proof of Lemma 2.1, it remains to prove its "if" part, which is the main concern in this subsection.

Let $G: \mathcal{M} \rightarrow H^{*}$ be a $\left(\mu_{1} \times \mu_{2}\right)$-continuous vector measure of bounded variation. We want to show that there exists a $\widetilde{g} \in L_{\mathcal{M}}^{1}\left(X_{1} ; L^{1}\left(X_{2} ; H^{*}\right)\right)$ such that

$$
G(E)=\int_{E} \tilde{g} d \mu_{1} d \mu_{2}, \quad \forall E \in \mathcal{M} .
$$

Firstly, we show that if $E_{0} \in \mathcal{M}$ has a positive $\left(\mu_{1} \times \mu_{2}\right)$-measure, then $G$ has a Bochner integrable Radon-Nikodým derivative on an $\mathcal{M}$-measurable set $B$ satisfying $B \subset E_{0}$ and $\left(\mu_{1} \times \mu_{2}\right)(B)>0$.

Denote by $|G|$ the variation of $G$, which is a scalar measure (see [7, Definition 4 and Proposition 9 of Chapter 1, pp. 2-3]). It is easy to see that $|G|$ is a $\left(\mu_{1} \times \mu_{2}\right)$-continuous 
$\mathbb{R}^{+}$-valued measure. Applying the Radon-Nikodým Theorem (to $|G|$ and $\mu_{1} \times \mu_{2}$ ), one can find an $\mathcal{M}$-measurable subset $B$ of $E_{0}$ and a positive integer $k$ such that $|G|(A) \leq$ $k\left(\mu_{1} \times \mu_{2}\right)(A)$ for all $A \in \mathcal{M}$ with $A \subset B$. Define a linear functional $\ell$ on the subspace $\mathcal{S}$ of simple functions in $L_{\mathcal{M}}^{p}\left(X_{1}, L^{q}\left(X_{2}, H\right)\right)$ as follows:

$$
\ell(f)=\sum_{i=1}^{n} G\left(E_{i} \cap B\right)\left(x_{i}\right),
$$

where

$$
f=\sum_{i=1}^{n} x_{i} I_{E_{i}}, \quad x_{i} \in H, 1 \leq i \leq n,
$$

with $\left\{E_{i} \mid 1 \leq i \leq n\right\}$ being a partition of $X_{1} \times X_{2}$. It follows that

$$
\begin{aligned}
|\ell(f)| & =\left|\sum_{i=1}^{n} G\left(E_{i} \cap B\right)\left(x_{i}\right)\right|=\left|\sum_{i=1}^{n} \frac{G\left(E_{i} \cap B\right)}{\left(\mu_{1} \times \mu_{2}\right)\left(E_{i} \cap B\right)}\left(\left(\mu_{1} \times \mu_{2}\right)\left(E_{i} \cap B\right) x_{i}\right)\right| \\
& \leq \sum_{i=1}^{n} k\left|\left(\mu_{1} \times \mu_{2}\right)\left(E_{i} \cap B\right) x_{i}\right| \leq k\|f\|_{L^{1}\left(X_{i} \times X_{2} ; H\right)} \\
& \leq k \mu_{1}\left(X_{1}\right)^{1 / p} \mu_{2}\left(X_{2}\right)^{1 / q}\|f\|_{L_{\mathcal{M}}^{p}\left(X_{1} ; L^{q}\left(X_{2} ; H\right)\right)} .
\end{aligned}
$$

Therefore $\ell$ is a bounded linear functional on $\mathcal{S}$. By the Hahn-Banach Theorem, it has a bounded linear extension to $L_{\mathcal{M}}^{p}\left(X_{1}, L^{q}\left(X_{2}, H\right)\right)$ (the extension is still denoted by $\ell$ ). Hence there exists a $g \in L_{\mathcal{M}}^{p^{\prime}}\left(X_{1}, L^{q^{\prime}}\left(X_{2}, H^{*}\right)\right)$ such that

$$
\ell(f)=\int_{X_{1} \times X_{2}}\langle f, g\rangle_{H, H^{*}} d \mu_{1} d \mu_{2}, \quad \forall f \in L_{\mathcal{M}}^{p}\left(X_{1}, L^{q}\left(X_{2}, H\right)\right) .
$$

We have

$$
G(E \cap B)(x)=\ell\left(x I_{E}\right)=\int_{E}\langle x, g\rangle_{H, H^{*}} d \mu_{1} d \mu_{2}, \quad \forall x \in H, E \in M .
$$

Since $g \in L_{\mathcal{M}}^{p^{\prime}}\left(X_{1}, L^{q^{\prime}}\left(X_{2}, H^{*}\right)\right)$ is Bochner integrable, we see that

$$
G(E \cap B)(x)=\left(\int_{E} g d \mu_{1} d \mu_{2}\right)(x), \quad \forall x \in H, E \in M .
$$

Consequently,

$$
G(E \cap B)=\int_{E} g d \mu_{1} d \mu_{2}, \quad \forall E \in \mathcal{M} .
$$

Noting that $B \in \mathcal{M}$, and therefore replacing $E$ in (2.11) by $E \cap B$, we see that

$$
G(E \cap B)=\int_{E \cap B} g d \mu_{1} d \mu_{2}, \quad \forall E \in \mathcal{M} .
$$


Now by the Exhaustion Lemma ([7, p. 70]), there exist a sequence $\left\{A_{n}\right\}_{n=1}^{\infty}$ of disjoint members of $\mathcal{M}$ such that $\bigcup_{n=1}^{\infty} A_{n}=X_{1} \times X_{2}$ and a sequence $\left\{g_{n}\right\}_{n=1}^{\infty}$ of Bochner integrable functions on $X_{1} \times X_{2}$ such that

$$
G\left(E \cap A_{n}\right)=\int_{E \cap A_{n}} g_{n} d \mu_{1} d \mu_{2}, \quad \forall E \in \mathcal{M}, n \in \mathbb{N} .
$$

Define $\widetilde{g}: X_{1} \times X_{2} \rightarrow H^{*}$ by $\widetilde{g}\left(x_{1}, x_{2}\right)=g_{n}\left(x_{1}, x_{2}\right)$ if $\left(x_{1}, x_{2}\right) \in A_{n}$. It is obvious that $\tilde{g}$ is $\left(\mu_{1} \times \mu_{2}\right)$-measurable. Moreover, for each $E \in \mathcal{M}$ and all $m \in \mathbb{N}$,

$$
G\left(E \cap \bigcup_{n=1}^{m} A_{n}\right)=\int_{E} \tilde{g} I_{\bigcup_{n=1}^{m} A_{n}} d \mu_{1} d \mu_{2}
$$

Consequently,

$$
G(E)=\lim _{m \rightarrow \infty} \int_{E} \widetilde{g} I_{\bigcup_{n=1}^{m} A_{n}} d \mu_{1} d \mu_{2}, \quad \forall E \in \mathcal{M} .
$$

For $h \in H^{* *}$, the variation of $|G(h)|$ satisfies

$$
|G(h)|\left(X_{1} \times X_{2}\right) \geq \lim _{m \rightarrow \infty} \int_{X_{1} \times X_{2}}\left|\langle h, \tilde{g}\rangle_{H^{* *}, H^{*}}\right| I_{\bigcup_{n=1}^{m} A_{n}} d \mu_{1} d \mu_{2} .
$$

Hence by the Monotone Convergence Theorem, $\langle h, \widetilde{g}\rangle_{H^{* *}, H^{*}} \in L_{\mathcal{M}}^{1}\left(X_{1} ; L^{1}\left(X_{2} ; \mathbb{R}\right)\right)$ for each $h \in H^{* *}$. If $E \in \mathcal{M}$ and $h \in H^{* *}$, from the Dominated Convergence Theorem we have

$$
\begin{aligned}
\langle h, G(E)\rangle_{H^{* *}, H^{*}} & =\lim _{m \rightarrow \infty} \int_{X_{1} \times X_{2}}\langle h, \widetilde{g}\rangle_{H^{* *}, H^{*}} I_{\bigcup_{n=1}^{m} A_{n}} d \mu_{1} d \mu_{2} \\
& =\int_{X_{1} \times X_{2}}\langle h, \widetilde{g}\rangle_{H^{* *}, H^{*}} d \mu_{1} d \mu_{2} .
\end{aligned}
$$

Therefore $\widetilde{g}$ is Pettis integrable and its Pettis integral P- $\int_{X_{1} \times X_{2}} \widetilde{g} d \mu_{1} d \mu_{2}$ equals $G(E)$ for each $E \in \mathcal{M}$. Since $|G|\left(X_{1} \times X_{2}\right)$ is finite, $\int_{X_{1} \times X_{2}}|\widetilde{g}|_{H^{*}} I_{\bigcup_{n=1}^{m}} A_{n} d \mu_{1} d \mu_{2} \leq$ $|G|\left(X_{1} \times X_{2}\right)$ for all $m \in \mathbb{N}$. By the Monotone Convergence Theorem, $|\widetilde{g}|_{H^{*}} \in$ $L_{\mathcal{M}}^{1}\left(X_{1} ; L^{1}\left(X_{2} ; \mathbb{R}\right)\right)$. Hence $\tilde{g}$ is Bochner integrable. Since the Pettis and Bochner integrals coincide whenever they coexist, we obtain (2.10), proving the Radon-Nikodým property of $H^{*}$ with respect to $\left(X_{1} \times X_{2}, \mathcal{M}, \mu_{1} \times \mu_{2}\right)$.

\subsection{A corollary of Lemma 2.1}

We now give an interesting corollary of Lemma 2.1. We first state the following.

Lemma 2.2. Let

$$
\mathcal{M}=\left\{A \in \mathcal{B}[0, T] \otimes \mathcal{F}_{T} \mid t \mapsto I_{A}(t, \cdot) \text { is } \mathbb{F} \text {-progressively measurable }\right\} .
$$


Then $\mathcal{M}$ is a sub- $\sigma$-field of $\mathcal{B}[0, T] \otimes \mathcal{F}_{T}$. Moreover, a process $\varphi:[0, T] \times \Omega \rightarrow H$ is $\mathbb{F}$-progressively measurable if and only if it is $\mathcal{M}$-measurable.

According to Lemmas 2.1 and 2.2, we have the following interesting corollary, whose proof is straightforward.

Corollary 2.3. Let $0<s \leq T$ and suppose $H^{*}$ has the Radon-Nikodým property with respect to $([0, T] \times \Omega, \mathcal{M}, m \times \mathbb{P})$ (where $m$ is the Lebesgue measure). Then the following identities hold:

$$
\left\{\begin{array}{l}
L_{\mathbb{F}}^{p}\left(\Omega ; L^{q}(0, s ; H)\right)^{*}=L_{\mathbb{F}}^{p^{\prime}}\left(\Omega ; L^{q^{\prime}}\left(0, s ; H^{*}\right)\right), \quad 1 \leq p, q<\infty . \\
L_{\mathbb{F}}^{q}\left(0, s ; L^{p}(\Omega ; H)\right)^{*}=L_{\mathbb{F}}^{q^{\prime}}\left(0, s ; L^{p^{\prime}}\left(\Omega ; H^{*}\right)\right),
\end{array}\right.
$$

This Riesz-type Representation Theorem will be very useful below.

Remark 2.4. It is easy to see that the conclusions in both Lemma 2.2 and Corollary 2.3 hold for any given filtration $\mathbb{F}$ (i.e., not necessarily for the natural filtration generated by the Brownian motion $\{W(t)\}_{t \geq 0}$ ), and also if one replaces the $\mathbb{F}$-progressive measurability by any other measurability requirement, for example, adapted, optional, predictable, etc.

We refer to [14] for an application of Corollary 2.3 in the study of null controllability of forward stochastic heat equations with one control. We will give more applications of this result in our forthcoming papers.

\section{Answers to Problems (E) and (R)}

In this section, we return to our complete filtered probability space $(\Omega, \mathcal{F}, \mathbb{F}, \mathbb{P})$ and give answers to Problems (E) and (R).

For any $p \in[1, \infty)$ and $0<s \leq T$, define an operator $\mathbb{L}_{s}: L_{\mathbb{F}}^{p}\left(\Omega ; L^{1}(0, s ; H)\right) \rightarrow$ $L_{\mathcal{F}_{s}}^{p}(\Omega ; H)$ by

$$
\mathbb{L}_{s}(u(\cdot))=\int_{0}^{s} u(t) d t, \quad \forall u(\cdot) \in L_{\mathbb{F}}^{p}\left(\Omega ; L^{1}(0, s ; H)\right) .
$$

Concerning Problem (E), noting that $L_{\mathbb{F}}^{1}\left(0, s ; L^{p}(\Omega ; H)\right) \subseteq L_{\mathbb{F}}^{p}\left(\Omega ; L^{1}(0, s ; H)\right)$, we give the following positive answer (which is a little stronger than the desired (1.6)):

Theorem 3.1. If $H^{*}$ has the Radon-Nikodým property, then

$$
\mathbb{L}_{s}\left(L_{\mathbb{F}}^{1}\left(0, s ; L^{p}(\Omega ; H)\right)\right)=L_{\mathcal{F}_{s}}^{p}(\Omega ; H) .
$$

Moreover, for each $\phi(\cdot, s) \in L_{\mathcal{F}_{s}}^{p}(\Omega ; H)$, there is a $\varsigma(\cdot, s) \in L_{\mathbb{F}}^{1}\left(0, s ; L^{p}(\Omega ; H)\right)$ such that

$$
\left\{\begin{array}{l}
\mathbb{L}_{S}(\varsigma(\cdot, s))=\phi(\cdot, s), \\
\|\varsigma(\cdot, s)\|_{L_{\mathbb{F}}^{1}\left(0, s ; L^{p}(\Omega ; H)\right)} \leq\|\phi(\cdot, s)\|_{L_{\mathbb{F}}^{1}\left(0, s ; L^{p}(\Omega ; H)\right)}
\end{array}\right.
$$

(In general, the above $\varsigma(\cdot, s)$ is NOT unique.) 
The result in Theorem 3.1 turns out to be sharp for $p \in(1, \infty)$. Indeed, we have the following result of negative nature.

Theorem 3.2. For any $p \in(1, \infty)$ and any $r \in(1, \infty]$,

$$
\mathbb{L}_{s}\left(L_{\mathbb{F}}^{r}\left(0, s ; L^{p}(\Omega ; H)\right)\right) \subsetneq L_{\mathcal{F}_{s}}^{p}(\Omega ; H) .
$$

Remark 3.3. (1) In [6, VI, 68, pp. 130-131] and [8], some Radon-Nikodým type theorems were established for real-valued or vector-valued processes with finite variation. However, it seems that none of these results could be applied to prove Theorem 3.1.

(2) Thanks to Remark 2.4, the conclusion in Theorem 3.1 holds for any given filtration $\mathbb{F}$; and one may replace the $\mathbb{F}$-progressive measurability by any other measurability requirement.

(3) We believe that (3.1) is sharp in the sense that, for any $r \in(1, \infty]$ and any $p \in$ $[1, \infty]$,

$$
\left\{\begin{array}{l}
\mathbb{L}_{s}\left(L_{\mathbb{F}}^{r}\left(0, s ; L^{p}(\Omega ; H)\right)\right) \subsetneq L_{\mathcal{F}_{s}}^{p}(\Omega ; H), \\
\mathbb{L}_{s}\left(L_{\mathbb{F}}^{p}\left(\Omega ; L^{r}(0, s ; H)\right)\right) \subsetneq L_{\mathcal{F}_{s}}^{p}(\Omega ; H) .
\end{array}\right.
$$

Theorem 3.2 shows that the first conclusion in (3.3) is true for $p \in(1, \infty)$, and that, noting (1.1), the second conclusion in (3.3) is true for $p \in(1, r] \cap(1, \infty)$. The general case is under our investigation. Note that the above can also be written as

$$
\mathbb{L}_{s}\left(\bigcup_{q>1} L_{\mathbb{F}}^{p}\left(\Omega ; L^{q}(0, s ; H)\right)\right) \subsetneq L_{\mathcal{F}_{s}}^{p}(\Omega ; H) .
$$

As a consequence of Theorem 3.1, our answer to Problem (R) is as follows:

Corollary 3.4. If $H$ is a Hilbert space, then for any $p \in[1, \infty)$, one can find a constant $C>0$ such that for any $\zeta(\cdot) \in L_{\mathbb{F}}^{p}\left(\Omega ; L^{2}(0, T ; H)\right)$, there is a $u(\cdot) \in L_{\mathbb{F}}^{1}\left(0, T ; L^{p}(\Omega ; H)\right)$ so that equality (1.7) holds and

$$
\|u(\cdot)\|_{L_{\mathbb{F}}^{1}\left(0, T ; L^{p}(\Omega ; H)\right)} \leq C\|\zeta(\cdot)\|_{L_{\mathbb{F}}^{p}\left(\Omega ; L^{2}(0, T ; H)\right)} \cdot
$$

Remark 3.5. By Remark 3.3(2), it is easy to see that the conclusion in Corollary 3.4 also holds for adapted, optional or predictable stochastic processes.

Corollary 3.4 shows the existence of the representation of Itô integrals by Lebesgue/ Bochner integrals. The corollary follows easily from Theorem 3.1 by applying the wellknown result that any Hilbert space has the Radon-Nikodým property (e.g., [7]) and also using the Burkholder-Davis-Gundy inequality for vector-valued stochastic processes (see [5, Theorem 5.4] and [16, Corollary 3.11]).

The rest of this section is devoted to proving Theorems 3.1-3.2. For this, besides Corollary 2.3, we need the following result concerning range inclusion for operators, which can be found in [19, Lemma 4.13, pp. 94-95 and Theorem 4.15, p. 97], for example. 
Lemma 3.6. Suppose $B_{X}$ and $B_{Z}$ are the open unit balls in Banach spaces $X$ and $Z$, respectively. Let $L: X \rightarrow Z$ be a bounded linear operator whose range is denoted by $\mathcal{R}(L)$, and whose adjoint operator is denoted by $L^{*}: Z^{*} \rightarrow X^{*}$. Then the following two conclusions hold:

(i) If $\mathcal{R}(L)=Z$, then there is a constant $C>0$ such that

$$
\left\|z^{*}\right\|_{Z^{*}} \leq C\left\|L^{*} z^{*}\right\|_{X^{*},}, \forall z^{*} \in Z^{*} .
$$

(ii) If (3.4) holds for some constant $C>0$, then

$$
B_{Z} \subset C L\left(B_{X}\right) \equiv\left\{C L x \mid x \in B_{X}\right\} .
$$

Remark 3.7. (1) Clearly, by Lemma 3.6, we see that $\mathcal{R}(L)=Z$ if and only if (3.4) holds for some constant $C>0$. But this lemma goes a little further than this. Indeed, the second conclusion of the lemma provides a "quantitative" characterization $B_{Z} \subset C L\left(B_{X}\right)$, which is more delicate than $\mathcal{R}(L)=Z$. We shall use this result essentially when we answer Problem (C) in the next section.

(2) One should compare Lemma 3.6 with the following general range inclusion result (e.g., [13, Lemma 2.4 in Chap. 7]): Let $X, Y$ and $Z$ be Banach spaces with $X$ being reflexive, and let $F: Y \rightarrow Z$ and $G: X \rightarrow Z$ be bounded linear operators. Then

$$
\left|F^{*} z^{*}\right|_{Y^{*}} \leq C\left|G^{*} z^{*}\right|_{X^{*}}, \forall z^{*} \in Z^{*} \text {, for some constant } C>0 \Leftrightarrow \mathcal{R}(F) \subseteq \mathcal{R}(G) .
$$

As shown in [1], the equivalence (3.5) may fail when $X$ is not reflexive. Nevertheless, when $F$ is surjective (in particular when $Y=Z$ and $F=I$, the identity operator, the case considered in Lemma 3.6), this equivalence remains true (even without the reflexivity assumption for $X$ ) (see [20, Theorem 1.2 and Remark 1.3]). We refer to [21] for further range inclusion results.

Further, we need the following property of Wiener integrals, a special case of Itô integrals with deterministic integrands (e.g., [12, Theorem 2.3.4 in Chapter 2, p. 11]).

Lemma 3.8. For each $0 \leq a<b \leq T$ and $f \in L^{2}(a, b)$ (which is a deterministic function, i.e., it does not depend on $\omega \in \Omega)$, the Wiener integral $\int_{a}^{b} f(t) d W(t)$ is a Gaussian random variable with mean 0 and variance $\int_{a}^{b}|f(t)|^{2} d t$.

We are now in a position to prove Theorems 3.1-3.2.

Proof of Theorem 3.1. It suffices to show (3.2). Since $L_{\mathbb{F}}^{1}\left(0, s ; L^{p}(\Omega ; H)\right) \subseteq$ $L_{\mathbb{F}}^{p}\left(\Omega ; L^{1}(0, s ; H)\right)$ (algebraically and topologically), the restriction of the operator

$$
\mathbb{L}_{s}: L_{\mathbb{F}}^{p}\left(\Omega ; L^{1}(0, s ; H)\right) \rightarrow L_{\mathcal{F}_{s}}^{p}(\Omega ; H)
$$

to $L_{\mathbb{F}}^{1}\left(0, s ; L^{p}(\Omega ; H)\right)$ is a bounded linear operator from $L_{\mathbb{F}}^{1}\left(0, s ; L^{p}(\Omega ; H)\right)$ to $L_{\mathcal{F}_{s}}^{p}(\Omega ; H)$ (for simplicity, we still denote it by $\mathbb{L}_{s}$ ). By Lemma 3.6(ii) and Corollary 2.3 , by a simple scaling, we see that the desired result (3.2) is a consequence of

$$
\left\|\mathbb{L}_{s}^{*} \eta\right\|_{L_{\mathbb{F}}^{\infty}\left(0, s ; L^{p^{\prime}}\left(\Omega ; H^{*}\right)\right)} \geq\|\eta\|_{L_{\mathcal{F}_{s}}^{p^{\prime}}\left(\Omega ; H^{*}\right)}, \quad \forall \eta \in L_{\mathcal{F}_{s}}^{p^{\prime}}\left(\Omega ; H^{*}\right) .
$$


In order to prove (3.6), let us first find the adjoint operator $\mathbb{L}_{s}^{*}$ of $\mathbb{L}_{s}$. For any $u(\cdot) \in$ $L_{\mathbb{F}}^{1}\left(0, s ; L^{p}(\Omega ; H)\right)$, and $\eta \in L_{\mathcal{F}_{s}}^{p}(\Omega ; H)^{*}=L_{\mathcal{F}_{s}}^{p^{\prime}}\left(\Omega ; H^{*}\right)$, we have

$$
\begin{aligned}
\left\langle\mathbb{L}_{s} u, \eta\right\rangle & =\mathbb{E}\left(\int_{0}^{s} u(t) d t, \eta\right)_{H, H^{*}}=\int_{0}^{s} \mathbb{E}(u(t), \eta)_{H, H^{*}} d t \\
& =\int_{0}^{s} \mathbb{E}\left(u(t), \mathbb{E}\left[\eta \mid \mathcal{F}_{t}\right]\right)_{H, H^{*}} d t=\left\langle u, \mathbb{L}_{s}^{*} \eta\right\rangle,
\end{aligned}
$$

which leads to the following representation of the adjoint operator $\mathbb{L}_{s}^{*}$ :

$$
\left\{\begin{array}{l}
\mathbb{L}_{s}^{*}: L_{\mathcal{F}_{s}}^{p^{\prime}}\left(\Omega ; H^{*}\right) \rightarrow L_{\mathbb{F}}^{1}\left(0, s ; L^{p}(\Omega ; H)\right)^{*}=L_{\mathbb{F}}^{\infty}\left(0, s ; L^{p^{\prime}}\left(\Omega ; H^{*}\right)\right), \\
\left(\mathbb{L}_{s}^{*} \eta\right)(t)=\mathbb{E}\left[\eta \mid \mathcal{F}_{t}\right], \quad t \in[0, s], \eta \in L_{\mathcal{F}_{s}}^{p^{\prime}}\left(\Omega ; H^{*}\right) .
\end{array}\right.
$$

Now, we let $p>1$. Making use of (3.7), we find that

$$
\begin{aligned}
\left\|\mathbb{L}_{s}^{*} \eta\right\|_{L_{\mathbb{F}}^{\infty}\left(0, s ; L^{p^{\prime}}\left(\Omega ; H^{*}\right)\right)} & =\left[\sup _{t \in[0, s]} \mathbb{E}\left|\mathbb{E}\left[\eta \mid \mathcal{F}_{t}\right]\right|_{H^{*}}^{p^{\prime}}\right]^{1 / p^{\prime}} \\
& \geq\left[\mathbb{E}\left|\mathbb{E}\left[\eta \mid \mathcal{F}_{s}\right]\right|_{H^{*}}^{p^{\prime}}\right]^{1 / p^{\prime}}=\left[\mathbb{E}|\eta|^{p^{\prime}}\right]^{1 / p^{\prime}}=\|\eta\|_{L_{\mathcal{F}_{s}}^{p^{\prime}}\left(\Omega ; H^{*}\right)}
\end{aligned}
$$

Therefore, (3.6) holds for $p>1$.

Next, for $p=1$, we have

$$
\begin{aligned}
\left\|\mathbb{L}_{s}^{*} \eta\right\|_{L_{\mathbb{F}}^{\infty}\left(\Omega ; L^{\infty}\left(0, s ; H^{*}\right)\right)} & =\underset{\omega \in \Omega}{\operatorname{ess} \sup } \sup _{t \in[0, s]}\left|\mathbb{E}\left[\eta \mid \mathcal{F}_{t}\right]\right|_{H^{*}} \\
& \geq \underset{\omega \in \Omega}{\operatorname{ess} \sup }\left|\mathbb{E}\left[\eta \mid \mathcal{F}_{S}\right]\right|_{H^{*}}=\underset{\omega \in \Omega}{\operatorname{ess} \sup }|\eta(\omega)|_{H^{*}}=\|\eta\|_{L_{\mathcal{F}_{S}}^{\infty}\left(\Omega ; H^{*}\right)} .
\end{aligned}
$$

This implies that our conclusion also holds for $p=1$.

Proof of Theorem 3.2. Noting (2.1), it suffices to prove Theorem 3.2 for $r \in(1, \infty)$. Towards a contradiction, assume that

$$
\mathbb{L}_{s}\left(L_{\mathbb{F}}^{r}\left(0, s ; L^{p}(\Omega ; H)\right)\right)=L_{\mathcal{F}_{s}}^{p}(\Omega ; H) \quad \text { for some } p, r \in(1, \infty) .
$$

Since $L_{\mathbb{F}}^{r}\left(0, s ; L^{p}(\Omega ; H)\right) \subseteq L_{\mathbb{F}}^{1}\left(0, s ; L^{p}(\Omega ; H)\right) \subseteq L_{\mathbb{F}}^{p}\left(\Omega ; L^{1}(0, s ; H)\right)$ (algebraically and topologically), the restriction of the operator

$$
\mathbb{L}_{s}: L_{\mathbb{F}}^{p}\left(\Omega ; L^{1}(0, s ; H)\right) \rightarrow L_{\mathcal{F}_{s}}^{p}(\Omega ; H)
$$

to $L_{\mathbb{F}}^{r}\left(0, s ; L^{p}(\Omega ; H)\right)$ is again a bounded linear operator from $L_{\mathbb{F}}^{r}\left(0, s ; L^{p}(\Omega ; H)\right)$ to $L_{\mathcal{F}_{s}}^{p}(\Omega ; H)$ (for simplicity, still denoted by $\mathbb{L}_{s}$ ). Similar to (3.7), the representation of the adjoint operator $\mathbb{L}_{s}^{*}$ is given as follows:

$$
\left\{\begin{array}{l}
\mathbb{L}_{s}^{*}: L_{\mathcal{F}_{s}}^{p^{\prime}}\left(\Omega ; H^{*}\right) \rightarrow L_{\mathbb{F}}^{r^{\prime}}\left(0, s ; L^{p^{\prime}}\left(\Omega ; H^{*}\right)\right), \\
\left(\mathbb{L}_{s}^{*} \eta\right)(t)=\mathbb{E}\left[\eta \mid \mathcal{F}_{t}\right], \quad t \in[0, s], \eta \in L_{\mathcal{F}_{s}}^{p^{\prime}}\left(\Omega ; H^{*}\right) .
\end{array}\right.
$$


By (3.8), using Lemma 3.6(i) and Corollary 2.3, we conclude that there exists a constant $C>0$ such that for any $\eta \in L_{\mathcal{F}_{s}}^{p^{\prime}}\left(\Omega ; H^{*}\right)$,

$$
\|\eta\|_{L_{\mathcal{F}_{s}}^{p^{\prime}}\left(\Omega ; H^{*}\right)} \leq C\left\|\mathbb{L}_{s}^{*} \eta\right\|_{L_{\mathbb{F}}^{r^{\prime}}\left(0, s ; L^{p^{\prime}}\left(\Omega ; H^{*}\right)\right)}
$$

where $r^{\prime}=r /(r-1)$.

Fix any $x_{0} \in H^{*}$ satisfying $\left|x_{0}\right|_{H^{*}}=1$ (which is independent of the time variable $t$ and the sample point $\omega$ ). Consider a sequence $\left\{\eta_{n}\right\}_{n=1}^{\infty}$ of random variables defined by

$$
\eta_{n}=\int_{0}^{s} e^{n t} d W(t) x_{0}, \quad n \in \mathbb{N} .
$$

It is obvious that $\eta_{n} \in L_{\mathcal{F}_{s}}^{p^{\prime}}\left(\Omega ; H^{*}\right)$ for any $n \in \mathbb{N}$. By Lemma 3.8 , the integral $\int_{0}^{s} e^{n t} d W(t)$ is a Gaussian random variable with mean 0 and variance $\frac{e^{2 n s}-1}{2 n}$. Hence,

$$
\begin{aligned}
{\left[\mathbb{E}\left|\int_{0}^{s} e^{n t} d W(t)\right|^{p^{\prime}}\right]^{1 / p^{\prime}} } & =\left[\int_{-\infty}^{\infty} \frac{\sqrt{n}|x|^{p^{\prime}}}{\sqrt{\left(e^{2 n s}-1\right) \pi}} e^{-\frac{n x^{2}}{e^{2 n s}-1}} d x\right]^{1 / p^{\prime}} \\
& =\left[\int_{-\infty}^{\infty}\left(\frac{e^{2 n s}-1}{n}\right)^{p^{\prime} / 2} \frac{|x|^{p^{\prime}}}{\sqrt{\pi}} e^{-x^{2}} d x\right]^{1 / p^{\prime}} \\
& =\left(\frac{1}{\sqrt{\pi}} \int_{-\infty}^{\infty}|x|^{p^{\prime}} e^{-x^{2}} d x\right)^{1 / p^{\prime}} \sqrt{\frac{e^{2 n s}-1}{n}}
\end{aligned}
$$

Now, by (3.11), it is easy to see that

$$
\begin{aligned}
\left\|\eta_{n}\right\|_{L_{\mathcal{F}_{s}}^{p^{\prime}}\left(\Omega ; H^{*}\right)} & =\left[\mathbb{E}\left|\int_{0}^{s} e^{n t} d W(t) x_{0}\right|^{p^{\prime}}\right]^{1 / p^{\prime}}=\left[\mathbb{E}\left|\int_{0}^{s} e^{n t} d W(t)\right|^{p^{\prime}}\right]^{1 / p^{\prime}} \\
& =\left(\frac{1}{\sqrt{\pi}} \int_{-\infty}^{\infty}|x|^{p^{\prime}} e^{-x^{2}} d x\right)^{1 / p^{\prime}} \sqrt{\frac{e^{2 n s}-1}{n}} .
\end{aligned}
$$

Using (3.11) again, we have

$$
\begin{aligned}
\left\|\mathbb{E}\left[\eta_{n} \mid \mathcal{F}_{t}\right]\right\|_{L_{\mathbb{F}}^{r^{\prime}}\left(0, s ; L^{p^{\prime}}\left(\Omega ; H^{*}\right)\right)} & =\left\{\int_{0}^{s}\left[\mathbb{E}\left|\int_{0}^{t} e^{n \tau} d W(\tau) x_{0}\right|^{p^{\prime}}\right]^{r^{\prime} / p^{\prime}} d t\right\}^{1 / r^{\prime}} \\
& =\left\{\int_{0}^{s}\left[\mathbb{E}\left|\int_{0}^{t} e^{n \tau} d W(\tau)\right|^{p^{\prime}}\right]^{r^{\prime} / p^{\prime}} d t\right\}^{1 / r^{\prime}} \\
& =\left\{\int_{0}^{s}\left[\left(\frac{1}{\sqrt{\pi}} \int_{-\infty}^{\infty}|x|^{p^{\prime}} e^{-x^{2}} d x\right)^{1 / p^{\prime}} \sqrt{\frac{e^{2 n t}-1}{n}}\right]^{r^{\prime}} d t\right\}^{1 / r^{\prime}} \\
& \leq \frac{1}{\sqrt{n}}\left(\frac{1}{\sqrt{\pi}} \int_{-\infty}^{\infty}|x|^{p^{\prime}} e^{-x^{2}} d x\right)^{1 / p^{\prime}}\left(\int_{0}^{s} e^{n r^{\prime} t} d t\right)^{1 / r^{\prime}} \\
& \leq \frac{1}{\sqrt{n}}\left(\frac{1}{\sqrt{\pi}} \int_{-\infty}^{\infty}|x|^{p^{\prime}} e^{-x^{2}} d x\right)^{1 / p^{\prime}} \frac{e^{n s}}{\left(n r^{\prime}\right)^{1 / r^{\prime}}} .
\end{aligned}
$$


From (3.12) and (3.13), it follows that

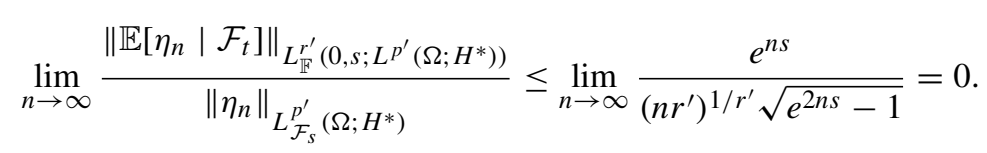

This, combined with (3.9), gives

$$
\lim _{n \rightarrow \infty} \frac{\left\|\mathbb{L}_{s}^{*} \eta_{n}\right\|_{L_{\mathbb{F}}^{r^{\prime}}\left(0, s ; L^{p^{\prime}}\left(\Omega ; H^{*}\right)\right)}}{\left\|\eta_{n}\right\|_{L_{\mathcal{F}_{s}}^{p^{\prime}}\left(\Omega ; H^{*}\right)}}=0
$$

which contradicts inequality (3.10). This completes the proof of Theorem 3.2.

\section{Answer to Problem (C)}

In this section we give a positive answer to Problem (C). Theorem 3.1 tells us that any Itô integral $\int_{0}^{s} \zeta(t) d W(t)$ with $\zeta(\cdot) \in L_{\mathbb{F}}^{p}\left(\Omega ; L^{2}(0, T ; H)\right)$ admits a (parameterized) Bochner integral representation, i.e. we can find a representor $u(\cdot, s) \in L_{\mathbb{F}}^{1}\left(0, s ; L^{p}(\Omega ; H)\right)$ (which is of course NOT unique) such that

$$
\int_{0}^{s} \zeta(t) d W(t)=\int_{0}^{s} u(t, s) d t, \quad \forall s \in[0, T]
$$

Put $Z \equiv L_{\mathbb{F}}^{1}\left(0, T ; L^{p}(\Omega ; H)\right)$. We now show that one can choose a $u(\cdot, s)$, which is continuous in $Z$ with respect to $s$, such that (4.1) holds. More precisely, we have the following result:

Theorem 4.1. For any given $\zeta(\cdot) \in L_{\mathbb{F}}^{p}\left(\Omega ; L^{2}(0, T ; H)\right.$ ), define a (set-valued) mapping $F:[0, T] \rightarrow 2^{Z}$ by

$$
F(s)=\left\{\eta(\cdot, s) \in Z \mid \int_{0}^{s} \eta(t, s) d t=\int_{0}^{s} \zeta(t) d W(t), \text { and } \eta(t, s)=0, \forall t>s\right\},
$$

Then $F$ has a continuous selection $f$.

Remark 4.2. If we choose $u(\cdot, s)$ to be the above $f(s)$, then $u(\cdot, s)$ is the desired process (for (4.1)), which is continuous in $Z$ with respect to $s$.

Before proving Theorem 4.1, we recall the following useful preliminary results.

Lemma 4.3. Let $X$ and $Y$ be two topological spaces. Then, for any set-valued mapping $\phi: X \rightarrow 2^{Y}$, the following two statements are equivalent:

(i) The map $\phi$ is lower semicontinuous, i.e., for any open subset $V$ of $Y$, the set $\{x \in X \mid$ $\phi(x) \cap V \neq \emptyset\}$ is open in $X$; 
(ii) If $x \in X, y \in \phi(x)$, and $V$ is a neighborhood of $y$ in $Y$, then there exists a neighborhood $U$ of $x$ in $X$ such that for every $x^{\prime} \in U$, there exists a $y^{\prime} \in \phi\left(x^{\prime}\right) \cap V$.

Lemma 4.4 ([15, Theorem 3.2" $]$ ). The following properties of a $T_{1}$-space are equivalent:

(i) $X$ is paracompact (i.e., any open cover of $X$ admits a locally finite open refinement, which is the case if $X$ is compact or is a metric space).

(ii) If $Y$ is a Banach space, then every lower semicontinuous mapping $F: X \rightarrow 2^{Y}$ such that $F(x)$ is a nonempty, closed, convex subset of $Y$ for any $x \in X$, admits a continuous selection, i.e., there exists a continuous mapping $f: X \rightarrow Y$ such that $f(x) \in F(x)$ for any $x \in X$.

We can now give a proof of Theorem 4.1.

Proof of Theorem 4.1. The main idea is to use Lemma 4.4. It is obvious that $[0, T]$ is a $T_{1}$-space and is paracompact. Hence we need only prove that $F(s)$ is a nonempty, closed, convex subset of $Z$ for any $s \in[0, T]$ and $F$ is lower semicontinuous. By Theorem 3.1, we see that $F(s)$ is nonempty. Also, it is very easy to check that $F(s)$ is a convex subset of $Z$ and is closed in $Z$.

It remains to show that $F$ is lower semicontinuous. Fix any $s \in[0, T]$, any $\eta(\cdot, s) \in$ $F(s)$, and any neighborhood $V$ of $\eta(\cdot, s)$ in $Z$. Clearly, there exists a $\delta>0$ such that

$$
V_{1}=\left\{z(\cdot) \in Z \mid\|z(\cdot)-\eta(\cdot, s)\|_{Z}<\delta\right\} \subset V .
$$

We claim that there exists an $\varepsilon>0$ such that for any $r$ satisfying $|r-s|<\varepsilon$,

$$
F(r) \cap V_{1} \neq \emptyset \text {. }
$$

This will yield the lower semicontinuity of $F(\cdot)$. To prove our claim, we first make use of the Burkholder-Davis-Gundy inequality for vector-valued stochastic process (see [5, Theorem 5.4] and [16, Corollary 3.11]) to get

$$
\mathbb{E}\left|\int_{r}^{s} \zeta(t) d W(t)\right|_{H}^{p} \leq \mathbb{E}\left[\sup _{r \leq h \leq s}\left|\int_{r}^{h} \zeta(t) d W(t)\right|_{H}^{p}\right] \leq C \mathbb{E}\left[\int_{r}^{s}|\zeta(t)|_{H}^{2} d t\right]^{p / 2} .
$$

Choose an increasing sequence $\left\{r_{k}\right\}_{k=1}^{\infty}$ such that $0 \leq r_{1} \leq r_{2} \leq \cdots \rightarrow s$. Since $\zeta(\cdot) \in$ $L_{\mathbb{F}}^{p}\left(\Omega ; L^{2}(0, T ; H)\right)$, by the Dominated Convergence Theorem we have

$$
\lim _{k \rightarrow \infty} \mathbb{E}\left[\int_{r_{k}}^{s}|\zeta(t)|_{H}^{2} d t\right]^{p / 2}=\lim _{k \rightarrow \infty} \mathbb{E}\left[\int_{0}^{T} I_{\left[r_{k}, s\right]}|\zeta(t)|_{H}^{2} d t\right]^{p / 2}=0 .
$$

Hence,

$$
\lim _{r \rightarrow s} \mathbb{E}\left[\int_{r}^{s}|\zeta(t)|_{H}^{2} d t\right]^{p / 2} \leq \lim _{k \rightarrow \infty} \mathbb{E}\left[\int_{r_{k}}^{s}|\zeta(t)|_{H}^{2} d t\right]^{p / 2}=0 .
$$

Therefore, it follows from (4.4) that there exists an $\varepsilon_{1}>0$ such that for any $0 \leq s-r$ $<\varepsilon_{1}$,

$$
\left\|\int_{r}^{s} \zeta(t) d W(t)\right\|_{L_{\mathcal{F}_{s}}^{p}(\Omega ; H)}<\delta / 3
$$


On the other hand, by the Hölder inequality and using the Dominated Convergence Theorem, similar to the proof of (4.5), we see that there exists an $\varepsilon_{2}>0$ (which may depend on $s$ ) such that for any $0 \leq s-r<\varepsilon_{2}$,

$$
\left\|\int_{r}^{s} \eta(t, s) d t\right\|_{L_{\mathcal{F}_{s}}^{p}(\Omega ; H)} \leq \int_{r}^{s}\|\eta(t, s)\|_{L_{\mathcal{F}_{s}}^{p}(\Omega ; H)} d t=\int_{r}^{s}\left[\mathbb{E}|\eta(t, s)|_{H}^{p}\right]^{1 / p} d t<\delta / 3 .
$$

Put $\varepsilon_{3}=\min \left\{\varepsilon_{1}, \varepsilon_{2}\right\}$. From (4.6)-(4.7) and noting that $\int_{0}^{s} \eta(t, s) d t=\int_{0}^{s} \zeta(t) d W(t)$, we conclude that for any $r$ with $0 \leq s-r<\varepsilon_{3}$,

$$
\begin{aligned}
\| \int_{0}^{r} \eta(t, s) d t-\int_{0}^{r} \zeta(t) & d W(t) \|_{L_{\mathcal{F}_{s}}^{p}(\Omega ; H)} \\
\leq & \left\|\int_{0}^{r} \eta(t, s) d t-\int_{0}^{s} \eta(t, s) d t\right\|_{L_{\mathcal{F}_{s}}^{p}(\Omega ; H)} \\
& +\left\|\int_{0}^{r} \zeta(t) d W(t)-\int_{0}^{s} \zeta(t) d W(t)\right\|_{L_{\mathcal{F}_{s}}^{p}(\Omega ; H)} \\
< & 2 \delta / 3 .
\end{aligned}
$$

By the second conclusion in Theorem 3.1 and (4.8), we see that there is a $\phi(\cdot, r) \in$ $L_{\mathbb{F}}^{1}\left(0, r ; L^{p}(\Omega ; H)\right)$ such that $\|\phi(\cdot, r)\|_{L_{\mathbb{F}}^{1}\left(0, r ; L^{p}(\Omega ; H)\right)}<2 \delta / 3$, and

$$
\int_{0}^{r} \phi(t, r) d t=\int_{0}^{r} \zeta(t) d W(t)-\int_{0}^{r} \eta(t, s) d t
$$

Put $\varrho(\cdot, r)=I_{[0, r]} \phi(\cdot, r)+I_{[0, r]} \eta(\cdot, s)$. It is obvious that $\varrho(\cdot, r) \in F(r)$, and

$$
\|\eta(\cdot, s)-\varrho(\cdot, r)\|_{L_{\mathbb{F}}^{1}\left(0, s ; L^{p}(\Omega, H)\right)} \leq \int_{r}^{s}\left[\mathbb{E}|\eta(t, s)|_{H}^{p}\right]^{1 / p} d t+\|\phi(\cdot, r)\|_{L_{\mathbb{F}}^{1}\left(0, r ; L^{p}(\Omega ; H)\right)}<\delta .
$$

Therefore, for any $0 \leq s-r<\varepsilon_{3}$, we have $\varrho(\cdot, r) \in V_{1}$, which gives (4.3). By a similar argument, one can show that there exists an $\varepsilon_{4}>0$ such that (4.3) holds for any $0 \leq r-s<\varepsilon_{4}$. Choosing $\varepsilon=\min \left\{\varepsilon_{3}, \varepsilon_{4}\right\}$, we see that (4.3) holds for any $|r-s|<\varepsilon$. By Lemma 4.3, we know that $F:[0, T] \rightarrow Z$ is lower semicontinuous.

Finally, thanks to Lemma 4.4, we conclude that there exists a continuous selection $f$ of $F$.

\section{Two illustrative applications}

In this section, we give two simple applications of our Theorems 3.1-3.2. More interesting and sophisticated applications will be presented in our forthcoming publications. 


\subsection{Application to the controllability problem}

Consider a one-dimensional controlled stochastic differential equation

$$
d x(t)=[b x(t)+u(t)] d t+\sigma d W(t),
$$

with $b$ and $\sigma$ being given constants. We say that system (5.1) is exactly controllable if for any $x_{0} \in \mathbb{R}$ and $x_{T} \in L_{\mathcal{F}_{T}}^{p}(\Omega ; \mathbb{R})$, there exists a control $u(\cdot) \in L_{\mathbb{F}}^{p}\left(\Omega ; L^{1}(0, T ; \mathbb{R})\right)$ such that the corresponding solution $x(\cdot)$ satisfies $x(0)=x_{0}$ and $x(T)=x_{T}$. By the variation of constants formula, we have

$$
x(T)=e^{b T} x_{0}+\int_{0}^{T} e^{b(T-t)} u(t) d t+\int_{0}^{T} e^{b(T-t)} \sigma d W(t) .
$$

Thus, exact controllability is equivalent to

$$
x_{T}-e^{b T} x_{0}-\int_{0}^{T} e^{b(T-t)} \sigma d W(t)=\int_{0}^{T} e^{b(T-t)} u(t) d t .
$$

Since $x_{T} \in L_{\mathcal{F}_{T}}^{p}(\Omega ; \mathbb{R})$, there exists a unique $\zeta(\cdot) \in L_{\mathbb{F}}^{p}\left(\Omega ; L^{2}(0, T ; \mathbb{R})\right)$ such that

$$
x_{T}=\mathbb{E} x_{T}+\int_{0}^{T} \zeta(t) d W(t) .
$$

Hence, to ensure (5.2), it suffices to have

$$
\mathbb{E} x_{T}-e^{b T} x_{0}+\int_{0}^{T}\left[\zeta(t)-e^{b(T-t)} \sigma\right] d W(t)=\int_{0}^{T} e^{b(T-t)} u(t) d t,
$$

which is guaranteed by Theorem 3.1. This means that (5.1) is exactly controllable.

On the other hand, surprisingly, in virtue of [18, Theorem 3.1], it is clear that system (5.1) is NOT exactly controllable if one restricts the admissible controls $u(\cdot)$ to be in $L_{\mathbb{F}}^{2}\left(\Omega ; L^{2}(0, T ; \mathbb{R})\right)$ ! Moreover, by Theorem 3.2 , we see that system $(5.1)$ is NOT exactly controllable either if one uses admissible controls $u(\cdot)$ in $L_{\mathbb{F}}^{2}\left(\Omega ; L^{q}(0, T ; \mathbb{R})\right)$ for any $q \in(1, \infty]$. This leads to a corrected formulation for the exact controllability of stochastic differential equations, as presented below.

A little more generally, we can consider the following multi-dimensional controlled linear stochastic differential equation:

$$
\left\{\begin{array}{l}
d y(t)=[A y(t)+B u(t)] d t+[C y(t)+D u(t)] d W(t), \quad 0 \leq t \leq T, \\
y(0)=y_{0} \in \mathbb{R}^{n}
\end{array}\right.
$$

where $A, C \in \mathbb{R}^{n \times n}$ and $B, D \in \mathbb{R}^{n \times m}(n, m \in \mathbb{N})$ are matrices. Various controllability issues for system (5.3) were studied, say, in [2, 3, 10, 18] and the references cited therein. Note however that, unlike the classical deterministic case, as far as we know, there exist no universally accepted notions of controllability in the stochastic setting so far. Inspired by the main results of this paper, we introduce the following definition. 
Definition 5.1. System (5.3) is said to be exactly controllable if for any $y_{0} \in \mathbb{R}^{n}$ and $y_{T} \in L_{\mathcal{F}_{T}}^{p}\left(\Omega ; \mathbb{R}^{n}\right)$, there exists a control $u(\cdot) \in L_{\mathbb{F}}^{p}\left(\Omega ; L^{1}\left(0, T ; \mathbb{R}^{m}\right)\right)$ such that $D u(\cdot) \in$ $L_{\mathbb{F}}^{p}\left(\Omega ; L^{2}\left(0, T ; \mathbb{R}^{n}\right)\right)$ and the corresponding solution $y(\cdot)$ of $(5.3)$ satisfies $y(T)=y_{T}$.

We need $D u(\cdot) \in L_{\mathbb{F}}^{p}\left(\Omega ; L^{2}\left(0, T ; \mathbb{R}^{n}\right)\right)$ in the above definition because it appears in the Itô integral $\int_{0}^{T}[C y(t)+D u(t)] d W(t)$. It is clear that, for the controllability of deterministic linear (time-invariant) ordinal differential equations, there is no difference between the controllability by using $L^{1}$ (in time) control and by using $L^{2}$ (or even analytic in time) control. However, our analysis above indicates that things are completely different in the stochastic setting. A detailed study of the controllability for system (5.3) (in the sense of Definition 5.1) seems to deviate from the theme of this paper, and therefore we shall address this topic in our forthcoming works.

Mimicking the above, we can also consider the more general case where the spaces $\mathbb{R}^{n}$ and $\mathbb{R}^{m}$ in system (5.3) and Definition 5.1 are replaced respectively by two Hilbert spaces $H_{1}$ and $H_{2}$, and the matrices $A, B, C$ and $D$ therein are replaced by suitable linear (maybe unbounded) operators. Definitely, such an extension will open the door to a systematic study of exact controllability of stochastic partial differential equations. This is actually one of the original motivations for the current paper. We hope that the results of this paper indeed pave the way to a better understanding of controllability problems for infinitedimensional stochastic control systems.

\subsection{Application to a Black-Scholes model}

Consider a Black-Scholes market model

$$
\left\{\begin{array}{l}
d x_{0}(t)=r X_{0}(t) d t \\
d x(t)(t)=b X(t) d t+\sigma X(t) d W(t),
\end{array}\right.
$$

with $r, b, \sigma$ being constants. Under conditions of self-financing, and no transaction costs, the investor's wealth process $Y(\cdot)$ satisfies the equation

$$
d Y(t)=[r Y(t)+(b-r) Z(t)] d t+\sigma Z(t) d W(t),
$$

where $Z(t)$ is the amount invested in the stock. For convenience, a European contingent claim with payoff at the maturity $T$ being $\xi \in L_{\mathcal{F}_{T}}^{p}(\Omega ; \mathbb{R})$ is identified with $\xi$. Any such $\xi$ is said to be replicatable if there exists a trading strategy $Z(\cdot)$ such that for some $Y_{0}$ (the price of the contingent claim at $t=0$ ), one has

$$
Y(0)=Y_{0}, \quad Y(T)=\xi .
$$

In other words, a contingent claim $\xi$ is replicatable if and only if the following backward stochastic differential equation (BSDE, for short) admits an adapted solution $(Y(\cdot), Z(\cdot))$ :

$$
\left\{\begin{array}{l}
d Y(t)=[r Y(t)+(b-r) Z(t)] d t+\sigma Z(t) d W(t), \quad t \in[0, T], \\
Y(T)=\xi
\end{array}\right.
$$


In this case, $Y(t)$ is the price of the contingent claim at time $t$. See [9] and [23] for some relevant details. Now, let us look at an extreme case,

$$
b-r>0, \quad \sigma=0 .
$$

In this case, $\xi$ is replicatable if and only if the following BSDE admits an adapted solution $(Y(\cdot), Z(\cdot))$ :

$$
\left\{\begin{array}{l}
d Y(t)=[r Y(t)+(b-r) Z(t)] d t, \quad t \in[0, T], \\
Y(T)=\xi .
\end{array}\right.
$$

Similar to the above subsection, we see that the above equation admits an adapted solution $(Y(\cdot), Z(\cdot))$, which means that $\xi$ is replicatable. Further, since $\xi$ is arbitrary, this also means that the market with conditions (5.4) is complete! This is a little surprising since $\sigma=0$ in the market model. Some further study along these lines will be carried out in our future publications.

Acknowledgments. This work is supported by the NSFC under grants 10831007,11101070 and 60974035, by the National Basic Research Program of China (973 Program) under grant 2011CB808002, by the Grant MTM2011-29306 of the MICINN, Spain, by NSF under grants DMS1007514 , and a key project from the NSF of China.

\section{References}

[1] Bouldin, R.: A counterexample in the factorization of Banach space operators. Proc. Amer. Math. Soc. 68, 327 (1978) Zbl 0377.47018 MR 0500202

[2] Buckdahn, R., Quincampoix, M., Tessitore, G.: A characterization of approximately controllable linear stochastic differential equations. In: Stochastic Partial Differential Equations and Applications-VII, Lect. Notes Pure Appl. Math. 245, Chapman \& Hall/CRC, Boca Raton, FL, 53-60 (2006) Zbl 1121.60061 MR 2227219

[3] Chen, S., Li, X., Peng, S., Yong, J.: On stochastic linear controlled systems. Unpublished manuscript

[4] Conway, J. B.: A Course in Functional Analysis. 2nd ed., Grad. Texts in Math. 96, Springer, New York (1990) Zbl 0706.46003 MR 1070713

[5] Cox, S., Veraar, M.: Vector-valued decoupling and the Burkholder-Davis-Gundy inequality. Illinois J. Math., in press; http://fa.its.tudelft.nl/ veraar/research/papers/Cox_Veraar_Decoupling.pdf

[6] Dellacherie, C., Meyer, P. A.: Probabilities and Potential B. North-Holland (1982) Zbl 0494.60002 MR 0745449

[7] Diestel, J., Uhl, Jr., J. J.: Vector Measures. Math. Surveys 15, Amer. Math. Soc., Providence, RI (1977) Zbl 0369.46039 MR 0453964

[8] Dinculeanu, N.: Vector-valued stochastic processes. II. A Radon-Nikodym theorem for vector-valued processes with finite variation. Proc. Amer. Math. Soc. 102, 393-401 (1988) Zbl 0669.60037 MR 0921006

[9] El Karoui, N., Peng, S., Quenez, M. C.: Backward stochastic differential equations in finance. Math. Finance 7, 1-71 (1997) Zbl 0884.90035 MR 1434407

[10] Goreac, A.: A Kalman-type condition for stochastic approximate controllability. C. R. Math. Acad. Sci. Paris 346, 183-188 (2008) Zbl 1139.60031 MR 2393638 
[11] Karatzas, I., Shreve, S. E.: Brownian Motion and Stochastic Calculus. Springer (1988) Zbl 0638.60065 MR 0917065

[12] Kuo, H. H.: Introduction to Stochastic Integration. Springer (2006) Zbl 1101.60001 MR 2180429

[13] Li, X., Yong, J.: Optimal Control Theory for Infinite-Dimensional Systems. Systems Control Found. Appl., Birkhäuser Boston, Boston, MA (1995) Z Zbl 0817.49001 MR 1312364

[14] Lü, Q.: Some results on the controllability of forward stochastic heat equations with control on the drift. J. Funct. Anal. 260, 832-851 (2011) Zbl 1213.60105 MR 2737398

[15] Michael, E.: Continuous selections I. Ann. of Math. 63, 361-382 (1956) Zbl 0071.15902 MR 0077107

[16] van Neerven, J. M. A. M., Veraar, M. C., Weis, L.: Stochastic integration in UMD Banach space. Ann. Probab. 35, 1438-1478 (2007) Zbl 1121.60060 MR 2330977

[17] Nualart, D.: The Malliavin Calculus and Related Topics. Springer (1995) Zbl 0837.60050 MR 1344217

[18] Peng, S.: Backward stochastic differential equation and exact controllability of stochastic control systems. Progr. Natur. Sci. (English Ed.) 4, 274-284 (1994) MR 1402663

[19] Rudin, W.: Functional Analysis. McGraw-Hill, New York (1973) Zbl 0253.46001 MR 0365062

[20] Vieru, A.: On null controllability of linear systems in Banach spaces. Systems Control Lett. 54, 331-337 (2005) Zbl 1129.93329 MR 2123706

[21] Wang, P., Zhang, X.: Range inclusion of operators on non-archimedean Banach space. Sci. China Math. 53, 3215-3224 (2010) Zbl 1218.46051 MR 2746318

[22] Yong, J.: Some problems related to the Black-Scholes type security markets. In: Stochastic Processes and Applications to Mathematical Finance, J. Akahori et al. (ed.), World Sci., Singapore, 369-400 (2004) MR 2202707

[23] Yong, J.: Completeness of security markets and solvability of linear backward stochastic differential equations. J. Math. Anal. Appl. 319, 333-356 (2006) Zbl 1092.60025 MR 2217865 